\title{
Characteristic classes of vector bundles with extra structure
}

Diplomarbeit von Alexander Rahm aus Wiesbaden angefertigt im Institut für Mathematik der

Georg-August-Universität zu Göttingen 2006, betreut von

Prof. Dr. Thomas Schick 


\section{Contents}

Introduction (please read first) . . . . . . . . . . . . 4

I Orientation and spin structure 5

\begin{tabular}{|lll}
\hline 1 & Reductions of the structure group & 7
\end{tabular}

$1.1 \quad H$-Reductions . . . . . . . . . . . . . . . . . . . . 7

Subgroup reduction theorem . . . . . . . . . . 8

1.2 Application on Riemannian manifolds . . . . . . . . . . . . . . 9

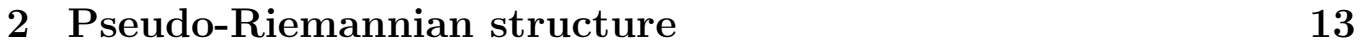

2.1 Pseudo-Riemannian metrics . . . . . . . . . . . . . . . . . . 13

2.2 Orientability of pseudo-Riemannian manifolds . . . . . . . . . 15

$2.3 \quad$ Pseudo-Riemannian Spin structures . . . . . . . . . . . . . . . 16

Pseudo-Riemannian product spin manifolds . . . . . . . . . . 18

The metric's influence on the existence of spin structures . . . 19

Elements of the proof of H. Baum's theorem . . . . . . . . . . 20

2.4 Review of a theorem by Frederik Witt . . . . . . . . . . . . . 24

$2.5 \quad$ Almost complex spin manifolds . . . . . . . . . . . . . . 26

II Complex structure 29

\begin{tabular}{|lll}
3 & Characteristic classes of "real bones" & 31
\end{tabular}

Motivation. . . . . . . . . . . . . . 31

General obstruction to "real bones" . . . . . . . . . . . . . . . 32

Basic requirement. . . . . . . . . . . . . . . . 33

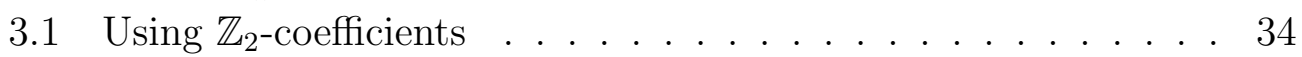

Classes fulfilling the basic requirement . . . . . . . . . 35

3.2 Using integral coefficients . . . . . . . . . . . . . . . . . . . . . 43

3.3 Conclusion . . . . . . . . . . . . . . . . . . . . 46 


\section{Introduction (please read first)}

This work is about properties of characteristic cohomology classes which occur when a special structure on vector bundles is given. I have focused on two different types of these structures, treated in two independent parts. The first part uses orientation and spin structure, and follows the literature in this area, mainly [Baum] and in the Riemannian section [Lawson \& Michelsohn], with some remarks. Two open questions are left in it, on pages 23 and 27. The second part uses complex structure and follows an idea on which I haven't found literature so far. The section 2.5 about almost complex spin manifolds on page 26 gives the link between the two parts. For the construction of the characteristic classes, I recommend the classic [Milnor \& Stasheff], as well as [Hatcher] and [Madsen \& Tornehave].

I'd like to thank my supervisor for lots of useful hints. 


\section{Part I}

Orientation and spin structure 



\section{Chapter 1}

\section{Reductions of the structure group}

Definition. Let $\lambda: H \rightarrow G$ be a homomorphism of topological groups, and let $P \rightarrow X$ be a principal $G$-bundle.

$(Q, f)$ shall be called a $\lambda$-reduction of $P$, if $Q \times H \stackrel{\bullet}{\longrightarrow} Q$

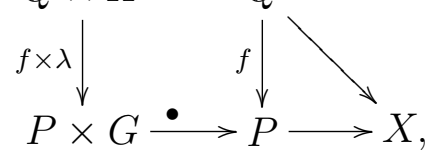

where $\bullet$ means the group operation, commutes, $\mathrm{f}$ is continuous and $Q \rightarrow X$ is a continuous principal $H$-bundle.

\section{$1.1 \quad H$-Reductions}

If the homomorphism $\lambda$ is the embedding of a subgroup $H$, one talks briefly about an $H$-reduction. There's a condition for the existence of such an $H$ reduction:

It's the existence of a global section s in the quotient bundle $P / H \rightarrow X$, which is obtained by dividing out the action of the subgroup $H$ in each fibre of $P \stackrel{p}{\longrightarrow} X$. Let the rest class map to this action be denoted by $\pi$. Given a continuous global section $s$, one gets a sub-bundle $Q^{(s)}$ of $P$ composed by the points whose image by $s$ of their projection into the base space $X$ is their rest class under the action of $H$ :

$$
Q^{(s)}:=\{z \in P \mid s(p(z))=\pi(z)\} .
$$


This means that the following diagram commutes:

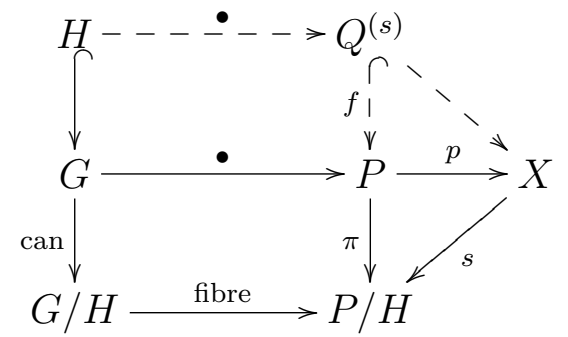

The bundle $Q^{(s)}$ admits as fibre an orbit of $H$, and that's why, according to the continuity of $s$, it's a principal $H$-bundle.

On the other hand, given an $H$-reduction $(Q, f)$ of $P$, the composition map $\pi \circ f$ is constant on the fibres of $Q_{b}, b \in X$. That's the case because the subgroup operation of $H$ on $P$ has the image $f\left(Q_{b}\right)$ as one orbit, as the upper left aisle of the diagram commutes. And thus the division by this operation puts $f\left(Q_{b}\right)$ into a single point. Therefore, it's possible to assign continuously to each point $b$ of the base space the constant image of the fibre $Q_{b}$, such as to obtain a global section $s_{(Q, f)}: X \rightarrow P / H$.

These reflections prove what I call the

\section{Subgroup reduction theorem}

Each global section in the quotient bundle corresponds in the described manner to an $H$-reduction of $P$.

Note 1. Every vector bundle admits the zero section as a global section. Presuming the base space to be a CW-complex, or especially a manifold, the existence of a global section is certainly preserved when forgetting the vector space structure, because the fibre remains contractible.

Note 2. Without proof. Let $K$ be a maximal compact Lie subgroup of $G$. Then $G / K$ is homeomorphic to a Euclidean space.

Example. $G L_{n}(\mathbb{R}) / \mathcal{O}_{n} \approx \mathbb{R}^{\frac{n(n+1)}{2}}$.

These two notes allow to deduce another theorem from the reduction theorem: 
Theorem 1. Let $K$ be a maximal compact Lie subgroup of $G$. Then any principal $G$-bundle $P \rightarrow X$ over a CW-complex $X$ admits a reduction to $K$.

Proof. Given a principal $G$-bundle $P \rightarrow X$, the quotient bundle $P / K \rightarrow$ $X$ admits as fibre a Euclidean space due to Note 2; and then after Note 1,

$P / K \rightarrow X$ admits a global section. Now the subgroup reduction theorem assures the existence of a $K$-reduction.

\subsection{Application on Riemannian manifolds}

Consider a real vector bundle $E \rightarrow M$ over a smooth connected Riemannian manifold $M$. As its fibre is $\mathbb{R}^{n}$, its structure group is $G L_{n}(\mathbb{R})$, and it can be obtained as associated bundle $E=P_{G L}(E) \times_{G L_{n}(\mathbb{R})} \mathbb{R}^{n}$ of the principal $G L_{n}(\mathbb{R})$-bundle $P_{G L}(E) \rightarrow M$.

In the local trivializations of $E$, each fibre $P_{G L}(E)_{b}$ is constructed as the Stiefel manifold $V_{n}$ consisting of the vector space bases of the corresponding fibre $E_{b}$. Then the trivialization changing maps of $P_{G L}(E)$ take the same values in $G L_{n}(\mathbb{R})$ as the corresponding maps of $E$, when identifying the elements $A \in G L_{n}(\mathbb{R})$ with the multiplication maps $(A \cdot): V_{n} \rightarrow V_{n}$,

$\left(e_{1}, \ldots, e_{n}\right) \mapsto\left(A e_{1}, \ldots, A e_{n}\right)$.

As $M$ can be provided with a CW-complex structure, and as $\mathcal{O}_{n}$ is a maximal compact Lie subgroup of $G L_{n}(\mathbb{R})$, theorem 1 assures the existence of an $\mathcal{O}_{n}$-reduction $P_{\mathcal{O}}(E)$ of $P_{G L}(E)$. Using the Riemannian metric on $M$, $P_{\mathcal{O}}(E)$ can be chosen to be the orthonormalization of $P_{G L}(E)$, each base $\left(e_{1}, \ldots, e_{n}\right)$ in a fibre of $P_{G L}(E)$ being projected to an orthonormal base by the Gram-Schmidt process.

$M$ could also be equipped with a Riemannian metric by choosing any smooth $\mathcal{O}_{n}$-reduction $P_{\mathcal{O}}(E)$ of $P_{G L}(E)$; calling orthonormal bases

all bases $\left(e_{1}, \ldots, e_{n}\right) \in P_{\mathcal{O}}(E)_{b} \subset P_{G L}(E)_{b}$, for every $b \in M$, and then inducing a norm by the vector space structure of $E_{b}$.

The question if $P_{\mathcal{O}}(E)$ in turn admits a reduction to $S \mathcal{O}_{n}$ is the question if $E$ is orientable. This can be seen by dividing the subgroup action of $S \mathcal{O}_{n}$ out of $P_{\mathcal{O}}(E)$ :

$P_{\mathcal{O}}(E) / S \mathcal{O}_{n}$ is called the orientation bundle of $E$, and is a principal $\mathbb{Z}_{2}$-bundle. An $S \mathcal{O}_{n}$-reduction $P_{S \mathcal{O}}(E) \subset P_{\mathcal{O}}(E)$ factors to a single sheet $P_{S \mathcal{O}}(E) / S \mathcal{O}_{n}$ of $P_{\mathcal{O}}(E) / S \mathcal{O}_{n}$ if it exists. Then the global section 


$$
M^{n} \stackrel{\cong}{\longrightarrow} P_{S \mathcal{O}}(E) / S \mathcal{O}_{n}
$$

into $P_{\mathcal{O}}(E) / S \mathcal{O}_{n}$ states the triviality of the orientation bundle. The rest class map to dividing out $S \mathcal{O}_{n}$ being continuous, now gives that $P_{\mathcal{O}}(E)$ like $P_{\mathcal{O}}(E) / S \mathcal{O}_{n}$ has two connected components ( $M$ was supposed connected). So, choosing an orientation of $E$ means choosing a connected component of $P_{\mathcal{O}}(E)$ as an $S \mathcal{O}_{n}$-reduction.

And this is possible if and only if the first Stiefel-Whitney class $w_{1}(E)$ vanishes.

\section{Why $E$ is orientable if and only if $w_{1}(E)=0$.}

I will take the sequence II.1.(1.2) from [Lawson \& Michelsohn, page 79]:

"Suppose $M$ connected. Then from the fibration $\mathcal{O}_{n} \longrightarrow P_{\mathcal{O}}(E) \longrightarrow M$, there is an exact sequence.

$$
0 \longrightarrow H^{0}\left(M, \mathbb{Z}_{2}\right) \longrightarrow H^{0}\left(P_{\mathcal{O}}(E), \mathbb{Z}_{2}\right) \stackrel{\bullet *}{\longrightarrow} H^{0}\left(\mathcal{O}_{n}, \mathbb{Z}_{2}\right) \stackrel{w_{E}}{\longrightarrow} H^{1}\left(M, \mathbb{Z}_{2}\right) \text {. }
$$

Contradiction. Take $E \rightarrow M$ to be the canonical $n$-plane bundle $\gamma^{n} \rightarrow$ $G_{n}$ over the infinite Grassmann manifold $G_{n}=G_{n}\left(\mathbb{R}^{\infty}\right)$. Theorem 7.1. of [Milnor \& Stasheff] yields $H^{0}\left(G_{n}, \mathbb{Z}_{2}\right)=\mathbb{Z}_{2}$, so $G_{n}$ meets the requirement to be connected. This theorem also gives $H^{1}\left(G_{n}, \mathbb{Z}_{2}\right) \approx\left\{0, w_{1}\left(\gamma^{n}\right)\right\} \approx \mathbb{Z}_{2}$. As $w_{1}\left(\gamma^{n}\right) \neq 0, \gamma^{n}$ is not orientable, and therefore $P_{\mathcal{O}}\left(\gamma^{n}\right)$ is connected. This means $H^{0}\left(P_{\mathcal{O}}\left(\gamma^{n}\right), \mathbb{Z}_{2}\right)=\mathbb{Z}_{2}$, so in the beginning of the exact sequence (1.2),

$$
0 \longrightarrow H^{0}\left(G_{n}, \mathbb{Z}_{2}\right) \stackrel{\pi^{*}}{\longrightarrow} H^{0}\left(P_{\mathcal{O}}\left(\gamma^{n}\right), \mathbb{Z}_{2}\right)
$$

$\pi^{*}$ is an isomorphism. Thus, $\bullet *$ must be zero, and therefore $w_{E}$ injective. As $w_{E}$ maps from $H^{0}\left(\mathcal{O}_{n}, \mathbb{Z}_{2}\right)=\mathbb{Z}_{2} \oplus \mathbb{Z}_{2}$ to $H^{1}\left(G_{n}, \mathbb{Z}_{2}\right)=\mathbb{Z}_{2}$, this is impossible.

Remedy. So, for the sequence (1.2) being exact, reduced cohomology theory ${ }^{1}$ has to be supposed. As $M$ is connected, so $\tilde{H}^{0}\left(M, \mathbb{Z}_{2}\right)=0$ and as $\tilde{H}^{0}\left(\mathcal{O}_{n}, \mathbb{Z}_{2}\right) \cong \mathbb{Z}_{2}$, the sequence then becomes

$$
0 \longrightarrow \tilde{H}^{0}\left(P_{\mathcal{O}}(E), \mathbb{Z}_{2}\right) \stackrel{\bullet *}{\longrightarrow} \mathbb{Z}_{2} \stackrel{w_{E}}{\longrightarrow} \tilde{H}^{1}\left(M, \mathbb{Z}_{2}\right)
$$

\footnotetext{
${ }^{1}$ I got this hint from V. Pidstrygach.
} 
If $E$ is orientable, $P_{\mathcal{O}}(E) / S \mathcal{O}_{n}$ is the trivial covering, so $P_{\mathcal{O}}(E)$ has two connected components. Then $\tilde{H}^{0}\left(P_{\mathcal{O}}(E), \mathbb{Z}_{2}\right) \cong \mathbb{Z}_{2}$, so for exactness $w_{E}=0$.

Else, if $E$ is not orientable, $P_{\mathcal{O}}(E) / S \mathcal{O}_{n}$ is connected, as well as $P_{\mathcal{O}}(E)$. Then $\tilde{H}^{0}\left(P_{\mathcal{O}}(E), \mathbb{Z}_{2}\right) \cong 0$, thus for exactness $w_{E}$ is injective, and $w_{E}(1) \neq 0$.

So $E$ is orientable if and only if $w_{E}(1)=0$. It can be shown, an argument is given by [Lawson \& Michelsohn], that $w_{E}(1)$ equals the first StiefelWhitney class $w_{1}(E)$.

Recall there's a two-fold covering homomorphism $\lambda: \operatorname{Spin}_{n} \rightarrow S \mathcal{O}_{n}$.

The existence of a Spin-structure on $E$ now means a further reduction, such that the following diagram commutes:

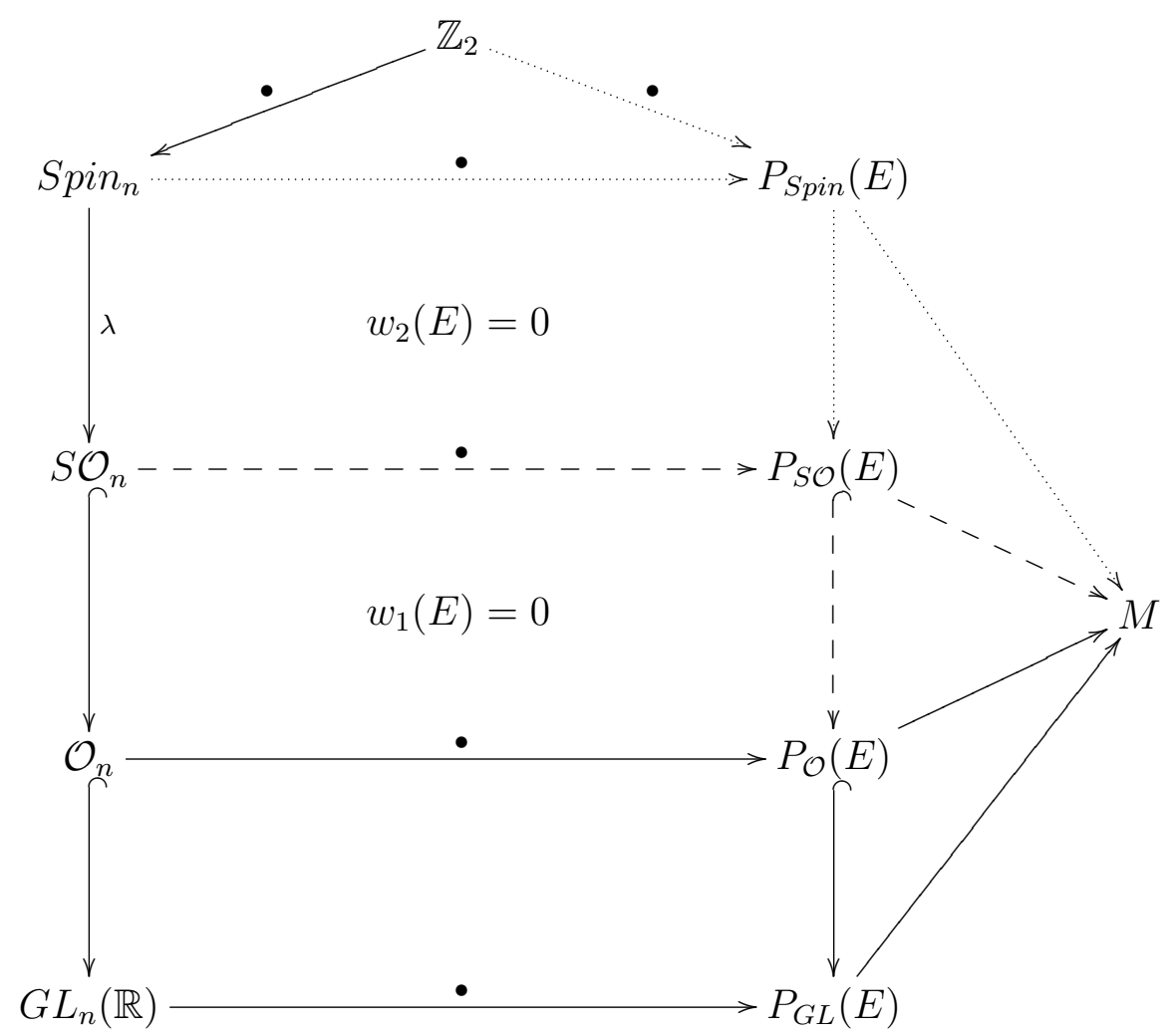

The same sequence reasoning as for the orientability applies for showing that the vanishing of the second Stiefel-Whitney class $w_{2}(E)$ means the existence of a $S p i n_{n}$-reduction of $P_{S \mathcal{O}}(E)$. Now note that $S \mathcal{O}_{n}$ is 0 -connected and for $n \geq 3$, Spin $_{n}$ is 1-connected. But the Serre spectral sequence can't be exploited any more to see if there's a relation between the vanishing of $w_{3}(E)$ and a reduction to a 2 -connected structure group. 


\section{Chapter 2}

\section{Pseudo-Riemannian structure}

\section{$2.1 \quad$ Pseudo-Riemannian metrics}

Let $M$ be a differentiable manifold of dimension $n$. A pseudo-Riemannian metric of index $k, 1 \leq k \leq n-1$, on $M$ is a smooth section

$g: M \rightarrow \mathrm{T}^{*} M \otimes \mathrm{T}^{*} M$, where $\mathrm{T}^{*} M$ is the dual of the tangent bundle $\mathrm{T} M$, such that for all $x \in M: g(x)$ is non-degenerated, symmetric and of index $k$.

Call $a_{1}, \ldots, a_{n}: U \rightarrow \mathrm{T} M$ a local pseudo-orthonormal frame,

if $g\left(a_{i}, a_{j}\right)=\chi(i) \delta_{i j}$, where $\chi(i)=\left\{\begin{array}{l}-1,1 \leq i \leq k \\ 1, \quad k<i \leq n\end{array}, \delta_{i j}=\left\{\begin{array}{l}1, i=j \\ 0, i \neq j\end{array}\right.\right.$,

and $U$ any open subset of $M$.

Let $e_{1}, \ldots, e_{n}=\left(\begin{array}{c}1 \\ 0 \\ \vdots \\ 0\end{array}\right), \ldots,\left(\begin{array}{c}0 \\ \vdots \\ 0 \\ 1\end{array}\right)$ denote the standard base of $\mathbb{R}^{n}$.

Now define a symmetric bilinear form on $\mathbb{R}^{n}$ by $<e_{i}, e_{j}>_{k}:=\chi(i) \delta_{i j}$. Fix the subgroup $\mathcal{O}_{(n, k)} \subset G L_{n}(\mathbb{R})$ of maps under which the bilinear form $<,>_{k}$ is unchanged. $\mathcal{O}_{(n, k)}$ is called the pseudo-orthogonal group of index $k$.

Theorem 2. There exists a pseudo-Riemannian metric of index $k$ on a smooth manifold $M$ of dimension $n$, if and only if the $n$-frame bundle $P_{G L}(\mathrm{~T} M)$ admits a reduction to the pseudo-orthogonal group $\mathcal{O}_{(n, k)}$.

Proof. Suppose that $P \rightarrow M$ is an $\mathcal{O}_{(n, k)}$-reduction of $P_{G L}(\mathrm{~T} M) \rightarrow M$. It 
follows from [Wolf, page 335, Lemma 11.1.5] that $\mathcal{O}_{k} \times \mathcal{O}_{n-k}$ is a maximal compact subgroup in $\mathcal{O}_{(n, k)}$. Lie group structure is transferred like in the footnote [on the same page]. Theorem 1 now provides an $\mathcal{O}_{k} \times \mathcal{O}_{n-k}$-reduction of $P$; call it $P^{\prime}$. The commutativity of the diagram

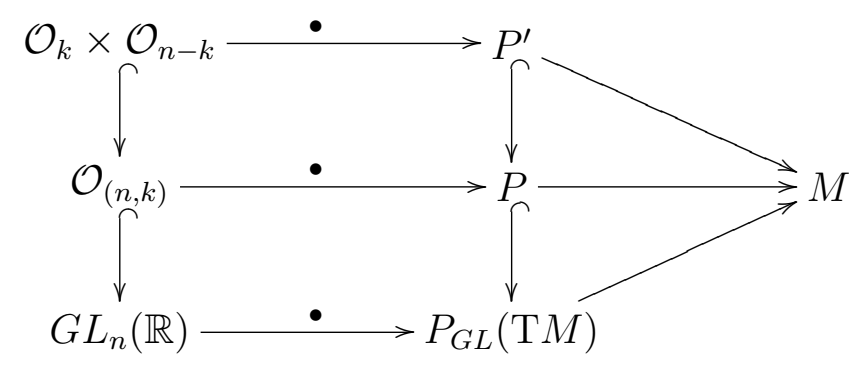

shows that $P^{\prime}$ is an $\mathcal{O}_{k} \times \mathcal{O}_{n-k^{-}}$reduction of $P_{G L}(\mathrm{~T} M) \rightarrow M$.

Denote by $\omega_{\mathcal{O}_{k}}$ the universal bundle over the classifying space $B \mathcal{O}_{k}$ for principal $\mathcal{O}_{k}$-bundles. According to [Husemoller, page 58, exercise 4.13.10], $B \mathcal{O}_{k} \times B \mathcal{O}_{n-k}$ is homotopy equivalent to $B\left(\mathcal{O}_{k} \times \mathcal{O}_{n-k}\right)$. Therefore, the classifying map of $P^{\prime}$ can be prolonged to $h_{P^{\prime}}: M \longrightarrow B \mathcal{O}_{k} \times B \mathcal{O}_{n-k}$.

(With this map, pull back $\omega_{\mathcal{O}_{k}} \times \omega_{\mathcal{O}_{n-k}}$, which is the universal bundle for principal $\left(\mathcal{O}_{k} \times \mathcal{O}_{n-k}\right)$-bundles [still stated by the last reference]). Writing $p r_{i}$ the projection on the $i$-th factor of the product $B \mathcal{O}_{k} \times B \mathcal{O}_{n-k}$, the bundle $P^{\prime}$ splits as the Whitney sum $P^{\prime}=P_{1} \oplus P_{2}$,

where $P_{1}=\left(p r_{1} \circ h_{P^{\prime}}\right)^{*} \omega_{\mathcal{O}_{k}}$, and $P_{2}=\left(p r_{2} \circ h_{P^{\prime}}\right)^{*} \omega_{\mathcal{O}_{n-k}}$.

As described in the application above, TM is the associated bundle $P_{G L}(\mathrm{~T} M) \times_{G L_{n}(\mathbb{R})} \mathbb{R}^{n}$. In terms of the reductions, this is

$$
\mathrm{T} M \cong P \times_{\mathcal{O}_{(n, k)}} \mathbb{R}^{n} \cong P^{\prime} \times_{\left(\mathcal{O}_{k} \times \mathcal{O}_{n-k}\right)} \mathbb{R}^{n} \cong\left(P_{1} \times_{\mathcal{O}_{k}} \mathbb{R}^{k}\right) \oplus\left(P_{2} \times_{\mathcal{O}_{n-k}} \mathbb{R}^{n-k}\right)
$$

Now choose Riemannian metrics $g_{1}$ on $P_{1} \times_{\mathcal{O}_{k}} \mathbb{R}^{k}$ and $g_{2}$ on $P_{2} \times_{\mathcal{O}_{n-k}} \mathbb{R}^{n-k}$. Then $\left(0, g_{2}\right)-\left(g_{1}, 0\right)$ is a pseudo-Riemannian metric of index $k$ on $M$.

Remark. The proof for the way back in [Baum, page 44, Satz 0.47] seems incomplete to me. I'll try to fill it up with some arguments; and declare the reduction $P^{\prime}$ slightly different.

Suppose that $M$ is equipped with a pseudo-Riemannian metric $g$ of index $k$. By the smoothness of $g$, its Eigen space $\xi_{x}$ to the Eigenvalue -1 in a fibre $\mathrm{T} M_{x}$ can only change smoothly from fibre to fibre. Thus, the bundle $\xi \rightarrow M$ of Eigen spaces to the Eigenvalue -1 is a differentiable sub-bundle of TM. In the same way, obtain the sub-bundle $\eta \subset \mathrm{T} M$ to the Eigenvalue 1. 
Now, every local pseudo-orthonormal $n$-frame $a_{1}, \ldots, a_{n}: M \supset U \rightarrow \mathrm{T} M$ provides a local $k$-frame $a_{1}, \ldots, a_{k}$ of $\xi$. As $g$ was assumed of index $k$, this is also the fibre dimension of $\xi$. Hence $a_{1}, \ldots, a_{k}$ is a local trivialization of $\xi$, and $a_{k+1}, \ldots, a_{n}$ one of $\eta$. This gives

$$
\mathrm{T} M=\xi \oplus \eta
$$

Note that $\xi$ has the property $g(\xi, \xi)<0$, which makes its vectors called timelike, whilst $\eta$ with $g(\eta, \eta)>0$ is called spacelike. This split into timeand space-sub-bundles enables to find an $\mathcal{O}_{k} \times \mathcal{O}_{n-k}$-reduction

$$
\begin{aligned}
& P^{\prime}:=\left\{\left(s_{1}, \ldots, s_{n}\right) \in P_{G L}(\mathrm{~T} M) \mid s_{1}, \ldots, s_{k} \epsilon \xi, s_{k+1}, \ldots, s_{n} \in \eta\right\} \\
& \text { of } P_{G L}(\mathrm{~T} M) . \text { It can be broadened to an } \mathcal{O}_{(n, k)} \text {-reduction. }
\end{aligned}
$$

\subsection{Orientability of pseudo-Riemannian manifolds}

It is described in [Wolf, page 341, first phrase], that the pseudo-orthogonal group $\mathcal{O}_{(n, k)}$ has four connected components, each containing one component of the maximal compact subgroup $\mathcal{O}_{k} \times \mathcal{O}_{n-k}$. Write

$$
\mathcal{O}_{(n, k)}^{++} \text {for the identity component, it contains } S \mathcal{O}_{k} \times S \mathcal{O}_{n-k}
$$

and label the others such that

$$
\begin{aligned}
& \mathcal{O}_{(n, k)}^{+-} \text {contains } S \mathcal{O}_{k} \times\left\{g \in \mathcal{O}_{n-k} \mid \operatorname{det} g=-1\right\}, \\
& \mathcal{O}_{(n, k)}^{-+} \text {contains }\left\{g \in \mathcal{O}_{k} \mid \operatorname{det} g=-1\right\} \times S \mathcal{O}_{n-k}, \text { and } \\
& \mathcal{O}_{(n, k)}^{--} \text {contains }\left\{g \in \mathcal{O}_{k} \mid \operatorname{det} g=-1\right\} \times\left\{g \in \mathcal{O}_{n-k} \mid \operatorname{det} g=-1\right\} .
\end{aligned}
$$

Call a smooth manifold $M$ with a pseudo-Riemannian metric of index $k$ a pseudo-Riemannian manifold. Let $M$ be of dimension $n$ and arcwise connected. Choose $G_{M}$ the group among the following subgroups of $\mathcal{O}_{(n, k)}$, 


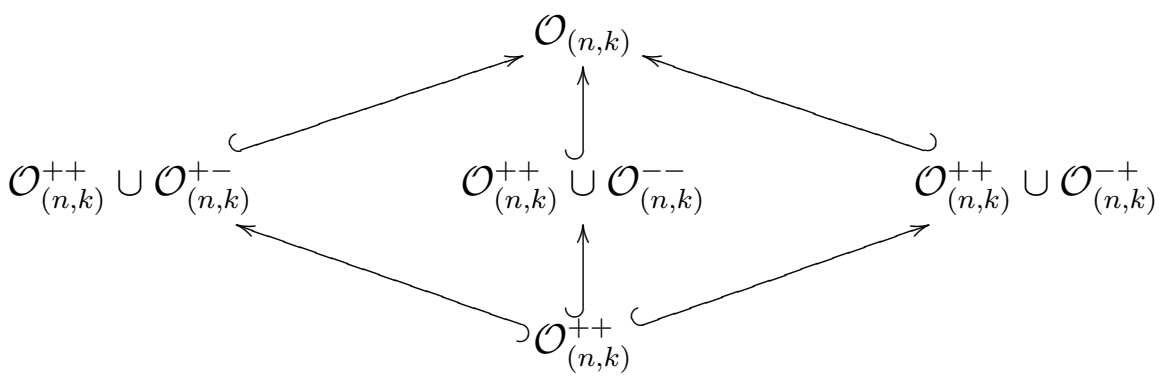

up to which the n-frame bundle $P_{G L}(\mathrm{~T} M)$ is reducible. For default, if $P_{\mathcal{O}_{(n, k)}}(\mathrm{T} M)$ admits no further reduction, choose $\mathcal{O}_{(n, k)}$ itself. This is the case when $P_{\mathcal{O}_{(n, k)}}(\mathrm{T} M)$ has just one connected component; $M$ is then without orientability. If $P_{\mathcal{O}_{(n, k)}}(\mathrm{T} M)$ has two components, the following three cases have to be distinguished:

$$
\begin{aligned}
G_{M} & =\mathcal{O}_{(n, k)}^{++} \cup \mathcal{O}_{(n, k)}^{+-}, M \text { time-orientable, } \\
G_{M} & =\mathcal{O}_{(n, k)}^{++} \cup \mathcal{O}_{(n, k)}^{-+}, M \text { space-orientable, } \\
G_{M} & =\mathcal{O}_{(n, k)}^{++} \cup \mathcal{O}_{(n, k)}^{--}, M \text { topologically orientable. }
\end{aligned}
$$

The last case is that $P_{\mathcal{O}_{(n, k)}}(\mathrm{T} M)$ has four components; then there's a reduction $P_{\mathcal{O}_{(n, k)}^{++}}(\mathrm{T} M)$, and $M$ is said to be with all types of orientability.

\subsection{Pseudo-Riemannian Spin structures}

Recall that the pseudo-orthogonal group $\mathcal{O}_{(n, k)}$ admits a two-fold covering $\operatorname{Pin}_{(n, k)}$ that arises in the Clifford algebra

$$
C l\left(\mathbb{R}^{n},<,>_{k}\right)=\sum_{r=0}^{\infty} \otimes^{r} \mathbb{R}^{n} /\left\langle x \otimes x+<x, x>_{k} 1\right\rangle_{x \in \mathbb{R}^{n}} \text { to the bilinear }
$$

form $<,>_{k}$. The covering map $\operatorname{Pin}_{(n, k)} \stackrel{\lambda}{\longrightarrow} \mathcal{O}_{(n, k)}$ here has the extra property to be a group homomorphism. Remember the notation on the page above and define $\tilde{G}_{M}:=\lambda^{-1}\left(G_{M}\right)$. As $G_{M}$ is a subgroup of $\mathcal{O}_{(n, k)}$ and $\lambda$ a group homomorphism, $\tilde{G}_{M}$ is a subgroup of $\operatorname{Pin}_{(n, k)}$. Therefore, observe the 
commutativity

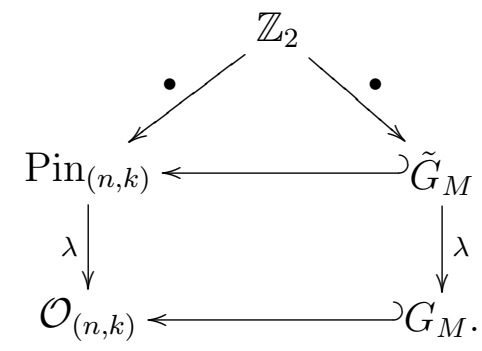

A spin structure on $M$ is now defined as a $\lambda$-reduction $(Q, f)$ of $P_{G_{M}}(\mathrm{~T} M)$, such that the following diagram commutes:

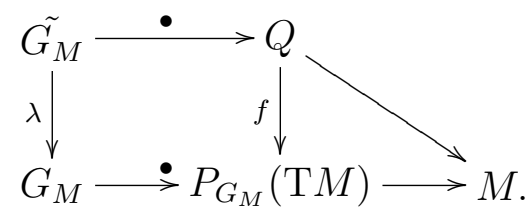

A pseudo-Riemannian manifold $M$ is called spin if it admits a spin structure. Recall from the proof of theorem 2, that the pseudo-Riemannian metric on $M$ induces a split T $M=\xi \oplus \eta$, into a timelike sub-bundle $\xi$ and a spacelike sub-bundle $\eta$.

\section{Theorem (H. Baum)}

Let $M$ be a smooth pseudo-Riemannian manifold. Then $M$ is spin if and only if the following condition on the Stiefel-Whitney classes holds:

$$
w_{2}(\mathrm{~T} M)=w_{1}(\xi) \cup w_{1}(\eta) .
$$

I won't give a full proof of Helga Baum's theorem, it is long and can be found in her book. Instead, I will note some consequences, and then show how the theorems exposed so far make their contributions to this proof. 


\section{Pseudo-Riemannian product spin manifolds}

Let $M_{1}$ and $M_{2}$ be smooth manifolds equipped with Riemannian metrics $r_{1}$ and $r_{2}$. The product manifold $M_{1} \times M_{2}$ with the pseudo-Riemannian metric $\left(-r_{1}, r_{2}\right)$ is spin if and only if its two factors admit spin structures as pseudo-Riemannian manifolds of index 0 .

This can be seen as follows: The metric $\left(-r_{1}, r_{2}\right)$ has time- and spacebundles $p r_{1}^{*} \mathrm{~T} M_{1}=\xi$ and $p r_{2}^{*} \mathrm{~T} M_{2}=\eta$. To

$w_{2}\left(\mathrm{~T}\left(M_{1} \times M_{2}\right)\right)=w_{2}\left(p r_{1}^{*} \mathrm{~T} M_{1} \oplus p r_{2}^{*} \mathrm{~T} M_{2}\right)$,

apply the Whitney sum axiom and get

$$
\begin{aligned}
& =w_{2}\left(p r_{1}^{*} \mathrm{~T} M_{1}\right)+w_{1}\left(p r_{1}^{*} \mathrm{~T} M_{1}\right) \cup w_{1}\left(p r_{2}^{*} \mathrm{~T} M_{2}\right)+w_{2}\left(p r_{2}^{*} \mathrm{~T} M_{2}\right) \\
& =p r_{1}^{*} w_{2}\left(\mathrm{~T} M_{1}\right)+p r_{2}^{*} w_{2}\left(\mathrm{~T} M_{2}\right)+w_{1}(\xi) \cup w_{1}(\eta) .
\end{aligned}
$$

So, the criterion of H. Baum's theorem for the existence of a spin structure on $M_{1} \times M_{2}$ is fulfilled, if and only if

$$
p r_{1}^{*} w_{2}\left(\mathrm{~T} M_{1}\right)+p r_{2}^{*} w_{2}\left(\mathrm{~T} M_{2}\right)=0
$$

As these pullbacks come from different base spaces, $w_{2}\left(\mathrm{~T} M_{1}\right)$ and $w_{2}\left(\mathrm{~T} M_{2}\right)$ must vanish independently.

If $M_{1}$ and $M_{2}$ are orientable, this is the case if and only if they both admit Riemannian spin structures.

Without knowing about the orientability of the two manifolds, a Riemannian spin structure can be considered as the oriented special case of a spin structure on a pseudo-Riemannian manifold of index 0 . The space bundle here is the full tangent bundle $\mathrm{T} M_{i}$. The time bundle then is the the trivial zero-dimensional bundle and has all Stiefel-Whitney classes zero, so the criterion in H. Baum's theorem for $M_{i}$ admitting a spin structure turns into $w_{2}\left(T M_{i}\right)=0$. And that's the condition obtained above. 


\section{The metric's influence on the existence of spin structures}

As reported in section 1.2 on page 11 , every Riemannian manifold admits a spin structure if and only if the second Stiefel-Whitney class of its tangential bundle vanishes. This is a purely topological criterion independent of the choice of metric. In contrast to this, we will now see that for a pseudoRiemannian manifold of dimension $n$ and index $1 \leq k \leq n-1$, the choice of metric does indeed matter for the existence of a spin structure.

Example. Consider $M=K^{2} \times K^{2}$, the product of two Klein bottles $K^{2}$. $K^{2}$ is non-orientable and admits a global nonzero vector field. Therefore, $w_{1}\left(\mathrm{~T} K^{2}\right) \neq 0$ and there's a trivial line-bundle $\varepsilon^{1}$ generated by the global section into $\mathrm{T} K^{2}$. Call $\kappa$ the orthogonal complement of $\varepsilon^{1}$ in $\mathrm{T} K^{2}$ with respect to a Riemannian metric on $K^{2}$. Then

$$
w_{2}\left(\mathrm{~T} K^{2}\right)=w_{2}\left(\varepsilon^{1} \oplus \kappa\right)=w_{2}\left(\varepsilon^{1}\right)+w_{1}\left(\varepsilon^{1}\right) \cup w_{1}(\kappa)+w_{2}(\kappa)=0 .
$$

Put a pseudo-Riemannian metric $g_{1}$ on $M$ determined by the time-bundle $\xi_{1}=p r_{1}^{*} \kappa$ and the space-bundle $\eta_{1}=p r_{1}^{*} \varepsilon^{1} \oplus p r_{2}^{*} \mathrm{~T} K^{2}$.

Use the Whitney sum axiom and naturality to compute

$$
\begin{aligned}
w_{2}(\mathrm{~T} M) & =w_{2}\left(p r_{1}^{*} \mathrm{~T} K^{2} \oplus p r_{2}^{*} \mathrm{~T} K^{2}\right) \\
& =p r_{1}^{*} w_{2}\left(\mathrm{~T} K^{2}\right)+w_{1}\left(p r_{1}^{*} \mathrm{~T} K^{2}\right) \cup w_{1}\left(p r_{2}^{*} \mathrm{~T} K^{2}\right)+p r_{2}^{*} w_{2}\left(\mathrm{~T} K^{2}\right)
\end{aligned}
$$

As stated above, $w_{2}\left(\mathrm{~T} K^{2}\right)=0$. So, the stability of Stiefel-Whitney classes under adding a trivial bundle makes the last term to

$$
=w_{1}\left(p r_{1}^{*} \kappa\right) \cup w_{1}\left(p r_{1}^{*} \varepsilon^{1} \oplus p r_{2}^{*} \mathrm{~T} K^{2}\right)=w_{1}\left(\xi_{1}\right) \cup w_{1}\left(\eta_{1}\right) .
$$

H. Baum's theorem now says that $M$ is spin.

The last term being a product of two non-zero elements over different base spaces, it is non-zero too. Equip $M$ with another metric $g_{2}$ by choosing as time- and space-bundles $\xi_{2}=p r_{1}^{*} \varepsilon^{1}$ and $\eta_{2}=p r_{1}^{*} \kappa \oplus p r_{2}^{*} \mathrm{~T} K^{2}$. Then

$$
w_{1}\left(\xi_{2}\right) \cup w_{1}\left(\eta_{2}\right)=0 \neq w_{1}\left(\xi_{1}\right) \cup w_{1}\left(\eta_{1}\right)=w_{2}(\mathrm{~T} M),
$$

and in this case, H. Baum's theorem says that $M$ is not spin. 


\section{Elements of the proof of H. Baum's theorem}

Let $K:=\mathcal{O}_{k} \times \mathcal{O}_{n-k}$ and $\tilde{K}:=\lambda^{-1}(K)$ be its pre-image under the covering homomorphism $\lambda$ :

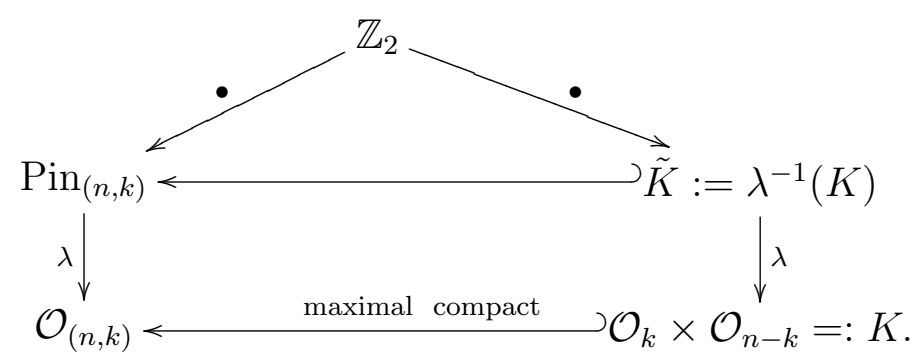

It follows from [Wolf, page 335, Lemma 11.1.5], that $K$ is a maximal compact subgroup in $\mathcal{O}_{(n, k)}$. Let $M$ be a pseudo-Riemannian manifold of dimension $n$ and index $k$. As described on page 15 , pick the group $G_{M}$ that matches with the orientability of $M$. Intersect the bottom line of the diagram with $G_{M}$. The intersection $K \cap G_{M}$ is maximal compact in $G_{M}$. Further, in the finite covering by $\lambda$,

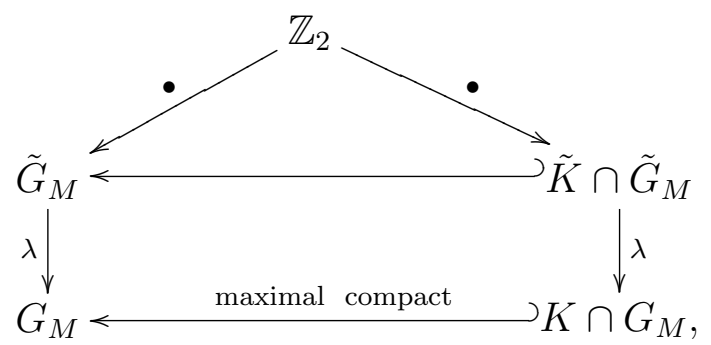

the pre-image $\lambda^{-1}\left(K \cap G_{M}\right)=\tilde{K} \cap \tilde{G}_{M}$ is in turn a maximal compact subgroup in $\tilde{G}_{M}$.

The following lemma inserts the split $\mathrm{T} M=\xi \oplus \eta$ into time- and spacebundle into the proof of $H$. Baum's theorem.

Applying theorem 1, there's a $K \cap G_{M}$-reduction $P^{\prime}$ of $P_{G_{M}}(\mathrm{~T} M)$.

Lemma. A spin structure on $M$ exists if and only if $P^{\prime}$ admits a $\lambda$ reduction.

Proof. Let $\left(Q^{\prime}, f^{\prime}\right)$ be a $\lambda$-reduction of $P^{\prime}$. Then

$Q:=Q^{\prime} \times_{\tilde{K} \cap \tilde{G}_{M}} \tilde{G}_{M}, f:=f^{\prime} \times \lambda$ is a $\lambda$-reduction of $P=P^{\prime} \times_{K \cap G_{M}} G_{M}$, hence a spin structure of $M$. 
On the other hand, let $(Q, f)$ be a spin structure of $M$. Then the following diagram commutes:

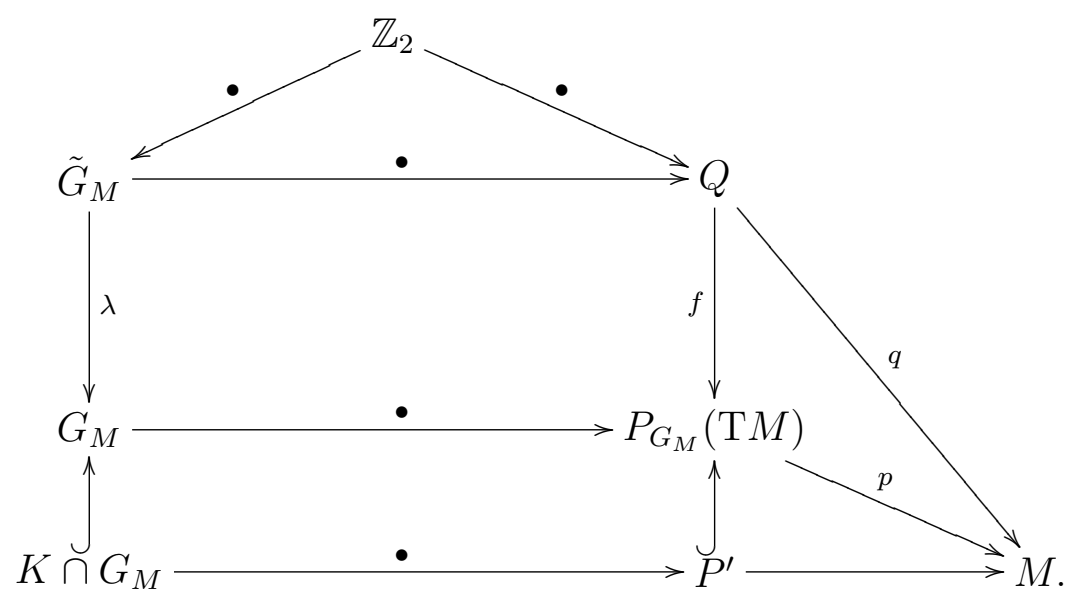

To the reduction $P^{\prime}$ of $P_{G_{M}}(\mathrm{~T} M)$, the subgroup reduction theorem (to be found on page 8) provides a global section

$\sigma: M \rightarrow P_{G_{M}}(\mathrm{~T} M) /\left(K \cap G_{M}\right)$. As the map $f$ induces a bijection

$f_{\pi}$ from $Q /\left(\tilde{K} \cap \tilde{G}_{M}\right)$ to $P_{G_{M}}(\mathrm{~T} M) /\left(K \cap G_{M}\right)$,

$\sigma$ is lifted to a global section $\tilde{\sigma}: M \rightarrow Q /\left(\tilde{K} \cap \tilde{G}_{M}\right)$ with $f_{\pi} \circ \tilde{\sigma}=\sigma$.

To $\tilde{\sigma}$, the subgroup reduction theorem donates a $\tilde{K} \cap \tilde{G}_{M^{-}}$-reduction $Q^{\prime}$ of $Q$. Let $f^{\prime}:=\left.f\right|_{Q^{\prime}}$. This looks like

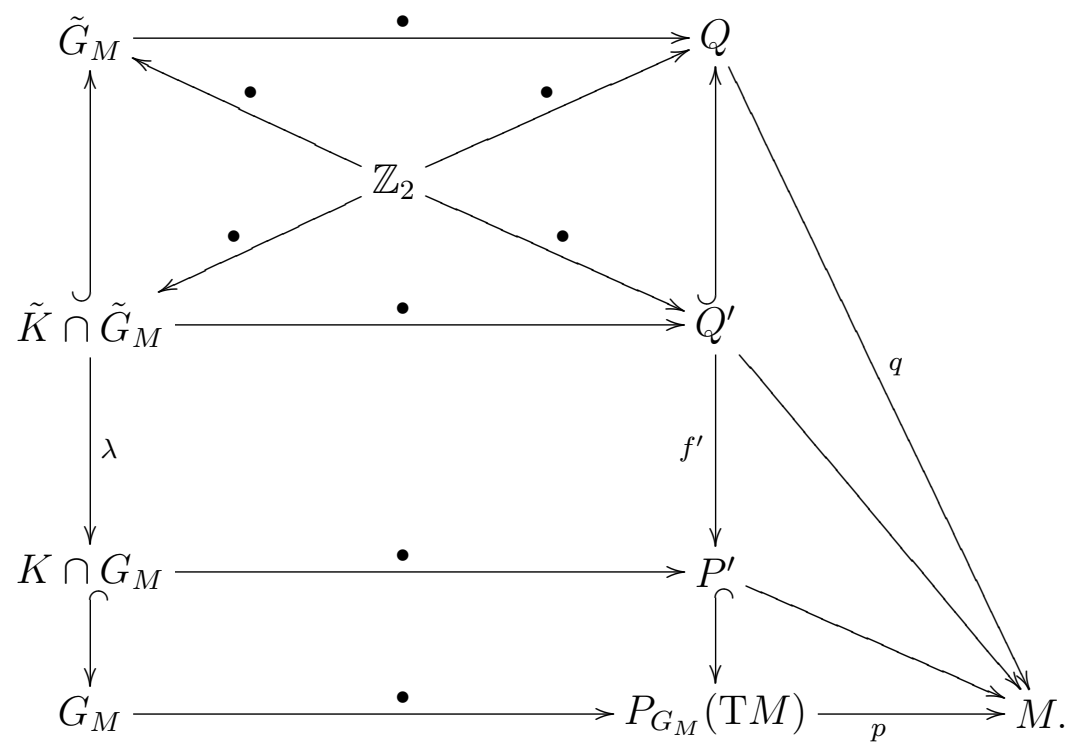


Let $\pi$ be the canonical rest class map from $P$ to $P /\left(K \cap G_{M}\right)$, and $\tilde{\pi}$ the one from $Q$ to $Q /\left(\tilde{K} \cap \tilde{G}_{M}\right)$. Because of $Q^{\prime}=\{y \epsilon Q \mid \tilde{\pi}(y)=\tilde{\sigma}(q(y))\}$, $P^{\prime}=\left\{x \in P_{G_{M}}(\mathrm{~T} M) \mid \pi(x)=\sigma(p(x))\right\},\left(Q^{\prime}, f^{\prime}\right)$ is a $\lambda$-reduction of $P^{\prime}$.

I'll begin now a diagram chase to verify that $f^{\prime}\left(Q^{\prime}\right)$ is indeed $P^{\prime}$. The reader, if bored, may skip this without inconvenience.

The situation is

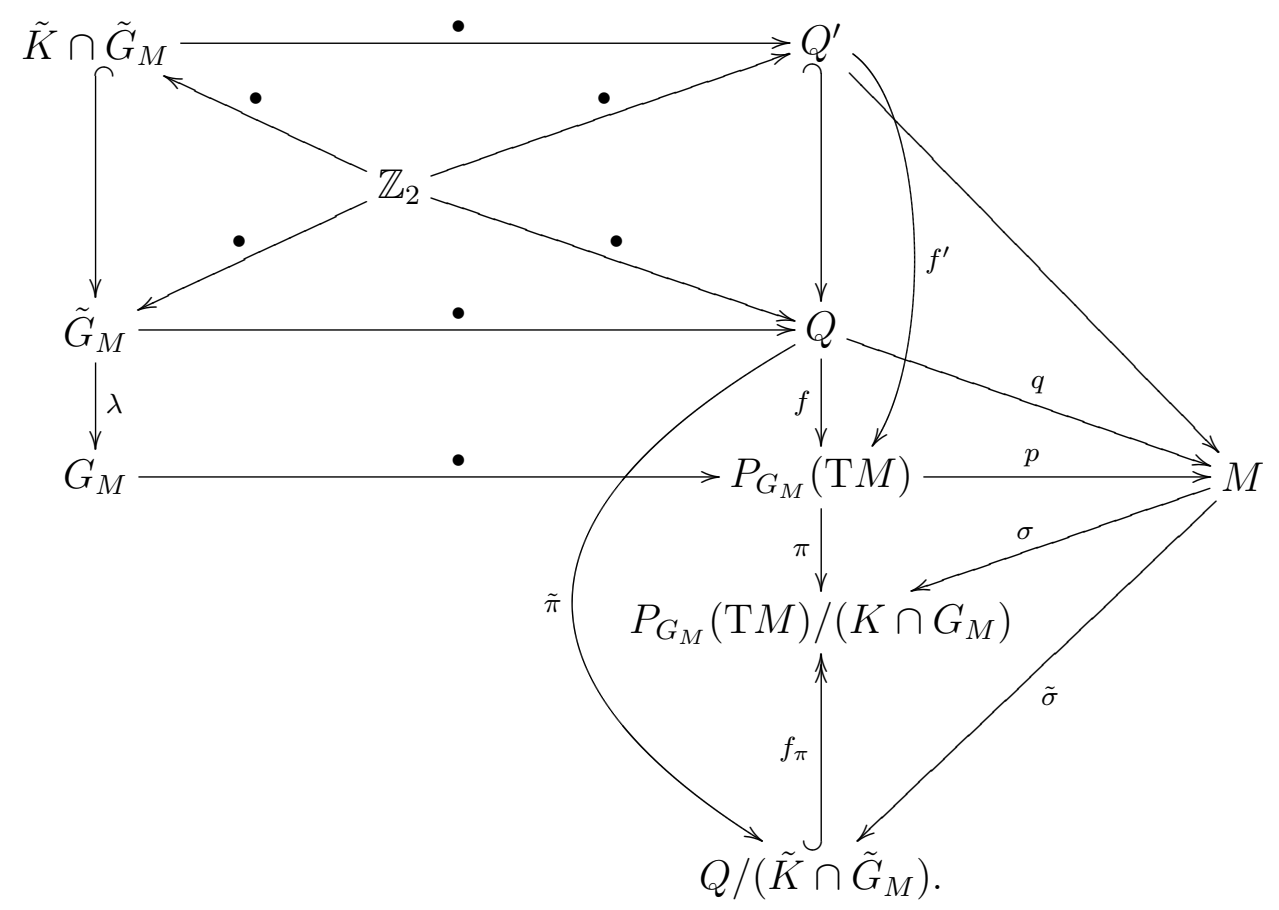

Let $y \in Q^{\prime}$. Then $\tilde{\pi}(y)=\tilde{\sigma}(q(y))$

$\Rightarrow f_{\pi}(\tilde{\pi}(y))=f_{\pi} \circ \tilde{\sigma}(q(y))=\sigma \circ q(y) \Rightarrow \pi \circ f(y)=\sigma \circ p \circ f(y)$

$\Rightarrow f(y) \in P^{\prime}$.

Let $x \in P^{\prime}$. As $f: Q \rightarrow P$ is a double covering, there are $y_{1}, y_{2} \in Q$ such that $f\left(y_{i}\right)=x$. Thus $\pi \circ f\left(y_{i}\right)=\sigma \circ p \circ f\left(y_{i}\right)$

$\Rightarrow \tilde{\pi}\left(y_{i}\right)=f_{\pi}^{-1} \circ \pi \circ f\left(y_{i}\right)={ }^{*} f_{\pi}^{-1} \circ \sigma \circ p \circ f\left(y_{i}\right)=\tilde{\sigma} \circ q\left(y_{i}\right) \Rightarrow y_{i} \epsilon Q^{\prime}$. 
The next element of the proof is based on obstructions against a global section in the bundle

$$
\overbrace{B\left(\tilde{K} \cap \tilde{G}_{M}\right) \stackrel{\rho(\lambda)}{\longrightarrow} B\left(K \cap G_{M}\right),}^{\mathcal{L}}
$$

where the classifying space $B G$ for principal $G$-bundles of any group $G$ is built with the Milnor Construction ${ }^{1}$. For any continuous group homomorphism $\lambda: H \rightarrow G$, the map $\rho(\lambda): B H \rightarrow B G$ is then defined by $[h, t] \mapsto[\lambda(h), t]$.

Theorem 0.33 .2 of [Baum, page 34 ] states that for the two-fold covering

$$
\lambda: \tilde{K} \cap \tilde{G}_{M} \rightarrow K \cap G_{M}
$$

$\mathcal{L}$ is a fibre bundle with fibre $B \mathbb{Z}_{2}$.

Contradiction. $\quad$ However, the fibre of $\rho(\lambda)$ over the point $\langle 1,1,0,0,0,0, \ldots\rangle \in B G$ consists of the single point $\langle 1,1,0,0,0,0, \ldots\rangle \in B H$ for any group homomorphism $\lambda: H \rightarrow G$.

$B \mathbb{Z}_{2}$ being not contractible, this contradiction remains under homotopy equivalence.

Remark. Some notion of functoriality seems to have motivated to map the fibre $\mathbb{Z}_{2}$ to of the principal bundle $\mathbb{Z}_{2} \stackrel{\bullet}{\longrightarrow} \tilde{K} \cap \tilde{G}_{M} \stackrel{\lambda}{\longrightarrow} K \cap G_{M}$ to the fibre $B \mathbb{Z}_{2}$. As seen, such a functoriality can't be achieved by the Milnor Construction.

Open question 1: Which classifying spaces for principal bundles can be used to save the statement?

I guess some affirmative solution to this question has already been published. Anyway, I couldn't find it so far.

1 Recall that the total space $E G$ in the Milnor Construction is obtained as the set $\left\{\left(g_{1}, t_{1}, g_{2}, t_{2}, g_{3}, t_{3} \ldots\right) \mid g_{i} \in G, t_{i} \in[0,1], \sum_{j \in \mathbb{N}} t_{j}=1, \quad t_{j}=0\right.$ for almost all $\left.j \in \mathbb{N}\right\}$, modulo the equivalence relation $\left(g_{1}, t_{1}, g_{2}, t_{2}, \ldots\right) \sim\left(g_{1}^{\prime}, t_{1}^{\prime}, g_{2}^{\prime}, t_{2}^{\prime}, \ldots\right) \Leftrightarrow \forall j \epsilon \mathbb{N}: t_{j}=t_{j}^{\prime}, g_{j}=g_{j}^{\prime}$ if $t_{j}=t_{j}^{\prime}>0$. The equivalence class $\left\langle g_{1}, t_{1}, g_{2}, t_{2}, \ldots\right\rangle$ is abbreviated by $[g, t]$. $E G$ is equipped with the smallest topology which makes the projections on the entries $t_{j}, g_{j}$ of $[g, t]$ continuous. The map $E G \times G \rightarrow E G,([g, t], a) \mapsto[g \cdot a, t]:=\left\langle g_{1} \cdot a, t_{1}, g_{2} \cdot a, t_{2}, \ldots\right\rangle$ defines a continuous right action of $G$ on $E G$, divided by which $B G$ is obtained. 


\subsection{Review of a theorem by Frederik Witt}

I'll translate theorem 2.2.9 of [Witt, page 16] and its proof into English and make some remarks.

Notations. Call a pseudo-Riemannian metric of index 1 a Lorentz metric. A nowhere vanishing global section into the one-dimensional time-bundle $\xi$ of a Lorentz metric determines an orientation of $\xi$ and is therefore called a time orientation.

Theorem. Let $M^{n}$ be a smooth manifold of dimension $n$. Then, the following conditions are equivalent:

(i) There exists a nowhere zero vector field on $M$.

(ii) $M$ is not compact, or $M$ is compact and its Euler number $\chi(M)$ is zero.

(iii) There exists a Lorentz metric on $M$.

(iv) There exists a time orientable Lorentz metric on $M$.

Proof.

(i) $\Leftrightarrow$ (ii) In the compact case, this is a classical result (theorem of Hopf): Look up [Bredon, corollary VII.14.5].

Remark 1. This corollary requires that $M$ is orientable. However, the general case is proved in [Alexandroff \& Hopf, pages 548-552, XIV 4, Satz III].

So let $M$ be a non compact manifold of dimension $n$. If $M$ is nonorientable, then $M$ can be replaced by a compact, orientable Lorentz manifold because the differential of the covering map is an isomorphism,

Remark 2. It is an isomorphism only locally.

so we can always switch to the case that $M$ is orientable.

Remark 3. This would ignore the problem of non-Orientability.

Then, due to obstruction-theoretic reflections, there exists a nowhere zero vector field on $M$ if and only if the Euler class $e(M) \in H^{n}(M, \mathbb{Z})$ vanishes. (For example, see corollary VII 14.4 in [Bredon] or [Milnor \& Stasheff, Chapter 12 , especially theorem 12.5$]$ ) 
Remark 4. The Euler class is only defined on oriented vector bundles. That's why these two references cannot be applied here in general.

But this vanishing follows directly from the well known result that

$H^{n}(M, \mathbb{Z})=0$ for non-compact manifolds

(look up [Stöcker \& Zieschang, example 13.6.6]).

Remark 5. For a manifold consisting only of non-compact connected components, it is known that a nowhere zero vector field exists. I'll only sketch the proof on one connected component:

There's always a vector field with finitely many singularities (points where it vanishes). In a connected non-compact differentiable manifold, there are arcs which connect the singularities with the "open rim". These arcs can be provided with tubular open neighborhoods, such that a new vector field can take the values of the old one, except in these tubular neighborhoods, where it takes just the values given on the borders of these tubes. Then the new vector field has no more singularities, they're "pushed out" through the "open rim".

(i) $\Rightarrow$ (iv) Choose a Riemannian metric $g$ on $M$. Let $T$ b a nowhere vanishing vector field; $T$ can be considered normalized with respect to $g$. Set $h:=g-T^{*} \otimes T^{*}$, where $T^{*}$ is the dual vector field to $T$, i.e., $T^{*}(Z)=g(T, Z)$ for all vector fields $Z$ on $M$.

Remark 6. $h$ must be set $h:=g-2 T^{*} \otimes T^{*}$.

There exist local vector fields $E_{2}, \ldots, E_{n}$ such that $\left(T, E_{2}, \ldots, E_{n}\right)$ are an orthonormal system with respect to g. Then

$h\left(E_{i}, E_{j}\right)=g\left(E_{i}, E_{j}\right)-2 g\left(T, E_{i}\right) g\left(T, E_{j}\right)=\delta_{j}^{i}, h\left(T, E_{j}\right)=g\left(T, E_{j}\right)=0$, and $h(T, T)=-1$; this means $h$ is a Lorentz metric with time orientation $T$.

(iv) $\Rightarrow$ (i) Choose the time orientation as a vector field.

(iv) $\Rightarrow$ (iii) obvious.

(iii) $\Rightarrow$ (ii) If $M$ is not compact, we're done. So let $M$ be compact, then we must show $\chi(M)=0$. If $h$ is time orientable, conclude (iv) $\Rightarrow(\mathrm{i}) \Rightarrow$ (ii). Otherwise consider the time orientation covering $\tilde{M}$ of $M$. As $M$ is compact, so is $\tilde{M}$, and we can use (iv) $\Rightarrow(\mathrm{i}) \Rightarrow$ (ii) to get $\chi(\tilde{M})=0$. This means $\chi(M)=\frac{1}{2} \chi(\tilde{M})=0$.

q.e.d. 


\subsection{Almost complex spin manifolds}

Definition. A smooth manifold $M$ of real dimension $2 m$ is called an

almost complex manifold, if its tangent bundle can be given a complex multiplication, such that it becomes a complex bundle $\zeta \rightarrow M$ of complex dimension $m$. Forgetting the complex structure, that's $\mathrm{T} M \cong \zeta_{\mathbb{R}}$.

Let $M$ be an almost complex manifold with a pseudo-Riemannian metric. Suppose further that $M$ is time- or space-orientable and $0=H^{2}(M, \mathbb{Z})$, which contains the first Chern class $c_{1}(\zeta)$. Then $M$ is spin, because H. Baum's theorem can be applied to

$$
w_{2}(\mathrm{~T} M)=w_{2}\left(\zeta_{\mathbb{R}}\right) \equiv c_{1}(\zeta) \bmod 2=0=w_{1}(\xi) \cup w_{1}(\eta),
$$

where the last equation is due to the orientability of $\xi$ or $\eta$.

This conclusion shall not be made without the almost complex structure on $M$.

Almost-example. Take the real projective space $\mathbb{R} P^{4 l+1}$. Frederik Witt's theorem can be applied to provide a time-orientable pseudo-Riemannian metric $g$ of index 1 , because $\mathbb{R} P^{4 l+1}$ is compact and its Euler characteristic $\chi\left(\mathbb{R} P^{4 l+1}\right)$ vanishes.

Lemma 4.3 in [Milnor \& Stasheff, page 42] gives $H^{2}\left(\mathbb{R} P^{4 l+1}, \mathbb{Z}_{2}\right)=\left\{0, a^{2}\right\}$,

where $a$ is the non-trivial element in $H^{1}\left(\mathbb{R} P^{4 l+1}, \mathbb{Z}_{2}\right) \cong \mathbb{Z}_{2}$ and generates

$H^{*}\left(\mathbb{R} P^{4 l+1}, \mathbb{Z}_{2}\right) \cong \mathbb{Z}_{2}[a] /\left\langle a^{4 l+2}=0\right\rangle$.

Theorem 4.5 of [Milnor \& Stasheff, page 45] yields

$w_{2}\left(\operatorname{TR} P^{4 l+1}\right)=\left(\begin{array}{c}4 l+2 \\ 2\end{array}\right) a^{2} \equiv a^{2} \bmod 2$. This differs from

$0=w_{1}(\xi) \cup w_{1}(\eta)$, which comes from the time-orientability of $g$.

So, $\mathbb{R} P^{4 l+1}$ with the time-orientable metric $g$ is not spin, in spite of

$H^{2}\left(\mathbb{R} P^{4 l+1}, \mathbb{Z}\right)=0$, compare [Baum, page 81 , last two lines]. 


\section{Counter-statement:}

For $l>0$, the last equation is contradicted by [Hatcher (AlgTop), page 214]: He calculates

$$
H^{*}\left(\mathbb{R} P^{4 l+1}, \mathbb{Z}\right) \approx \mathbb{Z}[\alpha, \beta] /\left(2 \alpha, \alpha^{2 l+1}, \beta^{2}, \alpha \beta\right),
$$

with $|\alpha|=2,|\beta|=4 l+1$.

Thus I get $H^{2}\left(\mathbb{R} P^{4 l+1}, \mathbb{Z}\right) \approx\{\alpha, 0\} \approx \mathbb{Z}_{2} \neq 0$.

For $l=0, H^{2}\left(\mathbb{R} P^{1}, \mathbb{Z}\right)=0$ is true, but also $H^{2}\left(\mathbb{R} P^{1}, \mathbb{Z}_{2}\right)=0$

$\Rightarrow w_{2}\left(\mathrm{TR} P^{1}\right)=0$.

Open question 2. Is there any smooth manifold $X$ with the desired properties of the almost-example, namely $H^{2}(X, \mathbb{Z})=0$, there exists a timeor space-orientable pseudo-Riemannian metric on $X$, and $w_{2}(\mathrm{~T} X) \neq 0$ ?

Take $X$ with $H^{2}(X, \mathbb{Z})=0$. I'll show why $w_{2}(\mathrm{~T} X)$ might however be non-zero.

Suppose $H^{1}(X, \mathbb{Z})=\mathbb{Z}_{2}$, then choose the free resolution

$$
0 \longrightarrow \mathbb{Z} \stackrel{\cdot 2}{\longrightarrow} \mathbb{Z} \longrightarrow \mathbb{Z}_{2} \longrightarrow 0
$$

Then [May, page 132, above the universal coefficient theorem] yields that

$0 \longrightarrow \operatorname{Hom}_{\mathbb{Z}}\left(\mathbb{Z}_{2}, \mathbb{Z}_{2}\right) \longrightarrow \operatorname{Hom}_{\mathbb{Z}}\left(\mathbb{Z}, \mathbb{Z}_{2}\right) \longrightarrow \operatorname{Hom}_{\mathbb{Z}}\left(\mathbb{Z}, \mathbb{Z}_{2}\right) \longrightarrow \operatorname{Ext}_{\mathbb{Z}}^{1}\left(\mathbb{Z}_{2}, \mathbb{Z}_{2}\right) \longrightarrow 0$

is exact. $\Rightarrow 0 \longrightarrow \mathbb{Z}_{2} \stackrel{\text { id }}{\longrightarrow} \mathbb{Z}_{2} \stackrel{0}{\longrightarrow} \mathbb{Z}_{2} \stackrel{\cong}{\longrightarrow} \operatorname{Ext}_{\mathbb{Z}}^{1}\left(\mathbb{Z}_{2}, \mathbb{Z}_{2}\right) \longrightarrow 0$.

$\Rightarrow \operatorname{Ext}_{\mathbb{Z}}^{1}\left(H^{1}(X, \mathbb{Z}), \mathbb{Z}_{2}\right)=\mathbb{Z}_{2}$. And using the universal coefficient theorem

$$
H^{2}\left(X, \mathbb{Z}_{2}\right)=\operatorname{Hom}_{\mathbb{Z}}\left(H_{2}(X, \mathbb{Z}), \mathbb{Z}_{2}\right) \oplus \operatorname{Ext}_{\mathbb{Z}}^{1}\left(H^{1}(X, \mathbb{Z}), \mathbb{Z}_{2}\right),
$$

I get $H^{2}\left(X, \mathbb{Z}_{2}\right)=\mathbb{Z}_{2}$. So, possibly $w_{2}(\mathrm{~T} X) \neq 0$. 
But it seems rather that a space $X$ with all these properties doesn't exist. That would mean that this attempt to use complex structure in order to obtain extra information about the underlying real bundle is failed. The following part II will in turn be an attempt to obtain supplementary information about complex bundles by some included real structure. 


\section{Part II}

\section{Complex structure}





\section{Chapter 3}

\section{Characteristic classes of "real bones"}

Definition. Consider a real vector bundle $F \rightarrow B$ and a complex vector bundle $E \rightarrow B$ over the same base space $B$.

If the fibre-wise constructed complexification $F \otimes_{\mathbb{R}} \mathbb{C}=: F^{\mathbb{C}}$ is isomorphic to $E$, I'll call $F$ a "real bone" bundle of $E$.

\section{Motivation.}

Not every complex vector bundle admits a "real bone" bundle. So, supplementary cohomological information might be gathered when restricting attention to the subcategory of complex vector bundles that admit one.

Example. Consider the canonical line bundle $\gamma_{1}\left(\mathbb{C}^{2}\right)$ over the onedimensional complex projective space $\mathbb{C} P^{1}$.

$\mathbb{C} P^{1}$ is homeomorphic to the real two-dimensional sphere $S^{2}$. Therefore, the real vector bundle $\gamma_{1}\left(\mathbb{C}^{2}\right)_{\mathbb{R}}$, obtained by forgetting the complex multiplication structure of $\gamma_{1}\left(\mathbb{C}^{2}\right)$, may be equipped with the base space $S^{2}$ :

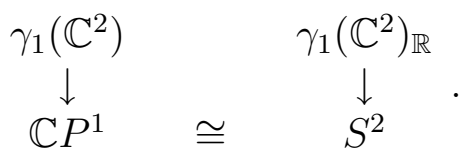

As $S^{2}$ is simply connected, every real line bundle over it must be orientable and hence trivial. This is especially the case for a susceptible "real bone" 
bundle $E$ of $\gamma_{1}\left(\mathbb{C}^{2}\right)$. But if $E^{\mathbb{C}} \cong \gamma_{1}\left(\mathbb{C}^{2}\right) \Rightarrow E \oplus E=\gamma_{1}\left(\mathbb{C}^{2}\right)_{\mathbb{R}}$, then the Euler class of $E$.

$e\left(\gamma_{1}\left(\mathbb{C}^{2}\right)_{\mathbb{R}}\right)=e(E \oplus E)=e(E) \cup e(E)$ would vanish due to the triviality

This is not the case, because $e\left(\gamma_{1}\left(\mathbb{C}^{2}\right)_{\mathbb{R}}\right)=c_{1}\left(\gamma_{1}\left(\mathbb{C}^{2}\right)\right)$, the top Chern class of $\gamma_{1}\left(\mathbb{C}^{2}\right)$, which is known to be nonzero.

Thus, there can't exist any "real bone" bundle of $\gamma_{1}\left(\mathbb{C}^{2}\right)$.

Another example. Consider the tangent bundle $T \mathbb{C} P^{1}$. This is not isomorphic $\left(^{1}\right.$ to $\gamma_{1}\left(\mathbb{C}^{2}\right)$.

Making use of a popular result, it can be instantly seen that $T \mathbb{C} P^{1}$ doesn't admit any "real bone" bundle, without having to deal with characteristic classes. Such a "real bone" bundle would be a smooth real line bundle splitting off from $\left(T \mathbb{C} P^{1}\right)_{\mathbb{R}} \cong T S^{2}$. Any orientation of this line bundle over the simply connected $S^{2}$ could be identified with a smooth, nowhere vanishing vector field. This is the impossible situation that "the hairy ball is combed". For the proof of this impossibility, see [Brouwer, page 112, Satz 2].

\section{General obstruction to "real bones"}

Consider a real vector bundle $F \rightarrow B$.

$F^{\mathbb{C}}$ has the complex multiplication $i(\vec{x}, \vec{y}):=(\vec{y},-\vec{x})$, whilst its conjugate bundle $\overline{F^{\mathbb{C}}}$ is equipped with $j(\vec{x}, \vec{y}):=(-\vec{y}, \vec{x})$ in every fibre. The reflection on the real axes - locally given by the orthonormal bases of the fibres of $F$-,

$r:\left(F^{\mathbb{C}}\right)_{\mathbb{R}} \rightarrow\left(\overline{F^{\mathbb{C}}}\right)_{\mathbb{R}},((\vec{x}, \vec{y}), b) \mapsto((\vec{x},-\vec{y}), b) \quad$ in the local trivializations

$\mathbb{R}^{2 n} \times U, U \subset B$, of $\left(F^{\mathbb{C}}\right)_{\mathbb{R}}=\left(\overline{F^{\mathbb{C}}}\right)_{\mathbb{R}}$, is an isomorphism of real bundles.

Involving the complex structure, $r: F^{\mathbb{C}} \rightarrow \overline{F^{\mathbb{C}}}$, is complex linear:

$\forall(z+i a) \in \mathbb{C},((\vec{x}, \vec{y}), b) \in F^{\mathbb{C}}:$

$r((z+i a)(\vec{x}, \vec{y}), b)=r((z \vec{x}, z \vec{y})+(a \vec{y},-a \vec{x}), b)=r((z \vec{x}+a \vec{y}, z \vec{y}-a \vec{x}), b)$

$=((z \vec{x}+a \vec{y},-z \vec{y}+a \vec{x}), b)=(z(\vec{x},-\vec{y})+a(\vec{y}, \vec{x}), b)=(z(\vec{x},-\vec{y})+a j(\vec{x},-\vec{y}), b)$

$=((z+j a)(\vec{x},-\vec{y}), b)=(z+j a) r((\vec{x}, \vec{y}), b)$.

${ }^{1}$ As $\mathbb{C} P^{1}$ is diffeomorphic to $S^{2},\left(T \mathbb{C} P^{1}\right)_{\mathbb{R}} \cong T S^{2}$.

$\Rightarrow w_{2}\left(T S^{2}\right) \equiv c_{1}\left(T \mathbb{C} P^{1}\right) \bmod 2$.

As the normal bundle $\nu S^{2}$ to $T S^{2}$ is trivial, $T S^{2}$ is stably trivial, hence

$w_{2}\left(T S^{2}\right)=w_{2}\left(T S^{2} \oplus \nu S^{2}\right)=w_{2}\left(\varepsilon^{3}\right)=0$.

$\Rightarrow c_{1}\left(T \mathbb{C} P^{1}\right) \equiv 0 \bmod 2$.

$\Rightarrow c_{1}\left(T \mathbb{C} P^{1}\right)$ doesn't generate $H^{*}\left(\mathbb{C} P^{1}, \mathbb{Z}\right)$ whilst $c_{1}\left(\gamma_{1}\left(\mathbb{C}^{2}\right)\right)$ generates $H^{*}\left(\mathbb{C} P^{1}, \mathbb{Z}\right)$ as a truncated polynomial ring [Milnor\&Stasheff, page 160 , theorem 14.4]. $\Rightarrow T \mathbb{C} P^{1} ¥ \gamma_{1}\left(\mathbb{C}^{2}\right)$. 
Hence $r$ is an isomorphism of complex vector bundles. So, any complex bundle $E \rightarrow B$ that admits $F$ as a "real bone" bundle must be isomorphic to its own conjugate bundle:

$$
E \cong F^{\mathbb{C}} \cong_{r} \overline{F^{\mathbb{C}}} \cong \bar{E} .
$$

This means for the odd Chern classes $c_{2 k+1}, k \in \mathbb{N} \cup\{0\}$, who have the property $c_{2 k+1}(E)=-c_{2 k+1}(\bar{E})$ (see [Milnor \& Stasheff, page 168, Lemma 14.9]), that

$$
c_{2 k+1}(E)=-c_{2 k+1}(\bar{E})=-c_{2 k+1}(E) \epsilon H^{4 k+2}(B, \mathbb{Z})
$$

$\Rightarrow c_{2 k+1}(E)=0$. Consequently, no complex vector bundle with some nonzero odd Chern class can admit a "real bone" bundle.

\section{A problem that occurs immediately:}

A complex vector bundle $E$ may admit different "real bone" bundles that are not bundle-isomorphic.

Example. Have a look at the cylinder and the Möbius band, considered as real line bundles over the circle. They're both "real bone" bundles of the trivial complex line bundle, but the first one is trivial and the second one is the non-trivial Möbius bundle.

Strategy. I want to attribute topological characteristic classes $c(F)$ of the "real bones" to the complexified bundles $F^{\mathbb{C}}$. For such an attribution being well-defined, I need that two "real bones" $F, G$ of the same complex bundle provide the same class $c(F)=c(G)$. For short, I get the

\section{Basic requirement.}

$F^{\mathbb{C}} \cong G^{\mathbb{C}} \Rightarrow c(F)=c(G)$. 


\subsection{Using $\mathbb{Z}_{2}$-coefficients}

I'll restrain myself to $\mathbb{Z}_{2}$-coefficients in the following.

Claim 1. A class $c$ fulfilling this basic requirement satisfies the

"Transferred stable invariance" property 2

$F^{\mathbb{C}} \cong B \times \mathbb{C}^{n} \Rightarrow c(F \oplus G)=c(G)$.

Proof. Let $c$ fulfill the basic requirement, and let $F^{\mathbb{C}} \cong B \times \mathbb{C}^{n}$. Let $G \rightarrow B$ be a real bundle.

Then $c(F \oplus G)=c\left(\left(B \times \mathbb{R}^{n}\right) \oplus G\right)$ because $\left(B \times \mathbb{R}^{n}\right)^{\mathbb{C}}=B \times \mathbb{C}^{n} \cong F^{\mathbb{C}}$, so the basic requirement can be applied.

As all real $n$-vector bundles can be pulled back from the universal bundle $\gamma_{n}\left(\mathbb{R}^{\infty}\right)$ over $B \mathcal{O}_{n}$, the fact [Hatcher, page 84, theorem 3.9] that $H^{*}\left(B \mathcal{O}_{n}, \mathbb{Z}_{2}\right)=$ $\mathbb{Z}_{2}\left[w_{1}\left(\gamma_{n}\left(\mathbb{R}^{\infty}\right)\right), \ldots, w_{n}\left(\gamma_{n}\left(\mathbb{R}^{\infty}\right)\right)\right]$ means that $c$ must be a polynomial $\sum \bigcup w_{i}$ in the Stiefel-Whitney classes. Thus, $c(F \oplus G)=\sum \bigcup w_{i}\left(\left(B \times \mathbb{R}^{n}\right) \oplus G\right)$ and with the stability [Hatcher, page 81] due to the Whitney-sum axiom of the Stiefel-Whitney classes, this term equals $\sum \bigcup w_{i}(G)=c(G)$.

q.e.d.

Now let the base space $B$ be compact Hausdorffian.

Claim 2. In this case, in return "transferred stable invariance" of $c$ provides the basic requirement.

Proof. Let $F \rightarrow B$,

$G \rightarrow B$ be real bundles with $F^{\mathbb{C}} \cong G^{\mathbb{C}}$. Forgetting the complex structure, that's $F \oplus F \cong G \oplus G$. As $B$ is compact Hausdorffian, there is an inverse bundle $F^{-1} \rightarrow B$, such that $F \oplus F^{-1} \cong B \times \mathbb{R}^{N}$.

As seen in the last proof, $c(F)=c\left(F \oplus\left(B \times \mathbb{R}^{N}\right)\right)$. And that's, in turn, $c\left(F \oplus F \oplus F^{-1}\right)=c\left(G \oplus G \oplus F^{-1}\right)$.

Now, $\left(G \oplus F^{-1}\right)^{\mathbb{C}}=G^{\mathbb{C}} \oplus\left(F^{-1}\right)^{\mathbb{C}} \cong F^{\mathbb{C}} \oplus\left(F^{-1}\right)^{\mathbb{C}}=\left(F \oplus\left(F^{-1}\right)\right)^{\mathbb{C}} \cong B \times \mathbb{C}^{n}$. That's why I can apply the "transferred stable invariance" and obtain

$c(F)=c\left(G \oplus\left(G \oplus F^{-1}\right)\right)=c(G)$.

q.e.d.

\footnotetext{
${ }^{2}$ I have chosen this name because of the invariance of Chern classes under stabilizing with trivial complex bundles. This invariance transfers to their mod 2 -reductions, giving them the described property.
} 


\section{Classes fulfilling the basic requirement}

Assertion 1. All elements of the polynomial sub-ring $\mathbb{Z}_{2}\left[w_{i}^{2}\right]_{i \in \mathbb{N} \cup\{0\}}$ satisfy the basic requirement.

Proof. Let $F \rightarrow B$,

$G \rightarrow B$ be real bundles with $F^{\mathbb{C}} \cong G^{\mathbb{C}}$. Forgetting the complex structure, that's $F \oplus F \cong G \oplus G$. A consequence of working in $\mathbb{Z}_{2}$-coefficients is that all terms that appear twice in a sum vanish, just like

$$
\sum_{k=1}^{2 i} w_{k} w_{2 i-k}=w_{i}^{2}
$$

Knowing these two facts, and the naturality of Stiefel-Whitney classes under bundle isomorphisms, I just need to apply the Whitney sum axiom to check that $w_{i}^{2}$ fulfills the basic requirement:

$$
\begin{aligned}
& w_{i}^{2}(F)=\sum_{k=1}^{2 i} w_{k}(F) w_{2 i-k}(F)=w_{2 i}(F \oplus F) \\
& =w_{2 i}(G \oplus G)=\sum_{k=1}^{2 i} w_{k}(G) w_{2 i-k}(G)=w_{i}^{2}(G) .
\end{aligned}
$$

This equation being valid for all $i \in \mathbb{N} \cup\{0\}$, it just remains to check polynomials $\sum \bigcup w_{i}^{2}$. And this has become now only a question of commuting brackets (they commute because $2=0$ in $\mathbb{Z}_{2}$-coefficients):

$$
\left(\sum \bigcup w_{i}^{2}\right)(F)=\sum \bigcup\left(w_{i}^{2}(F)\right)=\sum \bigcup\left(w_{i}^{2}(G)\right)=\left(\sum \bigcup w_{i}^{2}\right)(G) .
$$

Let $c$ be a polynomial in the Stiefel-Whitney classes $w_{i}$ with the "transferred stable invariance" property.

Assertion 2. $c$ is in the polynomial sub-ring $\mathbb{Z}_{2}\left[w_{i}^{2}\right]_{i \in \mathbb{N} \cup\{0\}}$.

Proof.

Put $B \mathcal{O}:=E U / \mathcal{O}$, via the inclusion $\mathcal{O} \subset U$ induced by the canonical inclusion $\mathbb{R} \subset \mathbb{C}$.

According to [Cartan, page 17-22, mark (76)], there then is the Hopf spaces fibration

$$
U / \mathcal{O} \longrightarrow \mathrm{f} \longrightarrow \mathrm{O} \longrightarrow \mathrm{BU},
$$

where the projection $p$ is the rest class map to dividing the whole group $U$ out of $E U$; and $f: U / \mathcal{O} \rightarrow B \mathcal{O}$ embeds a fibre.

It is known since [Cartan, page 17-13, mark (46)], and summarized in [Bunke \& Schick, page 48, theorem B.2], that

$H^{*}\left(B \mathcal{O}, \mathbb{Z}_{2}\right)=\mathbb{Z}_{2}\left[\omega_{1}, \omega_{2}, \ldots\right]$ is the polynomial algebra with generators the Stiefel-Whitney classes 
$\omega_{i}:=w_{i}\left(\gamma\left(\mathbb{R}^{\infty}\right)\right)$ of the universal real bundle over $B \mathcal{O}$.

Henri Cartan [page 17-22, mark (76)] has shown that $f^{*}$ maps these generators $\omega_{i}$ to the generators $v_{i}:=w_{i}\left(f^{*} \gamma\left(\mathbb{R}^{\infty}\right)\right)$ of the exterior algebra

$H^{*}\left(U / \mathcal{O}, \mathbb{Z}_{2}\right)=\bigwedge\left(\mathbb{Z}_{2}\left[v_{1}, v_{2}, \ldots\right]\right)$, which is obtained by dividing the ideal $\left\langle v_{i}^{2}\right\rangle_{i \in \mathbb{N} \backslash\{0\}}$ out of the polynomial algebra $\mathbb{Z}_{2}\left[v_{1}, v_{2}, \ldots\right]$. Hence, exactly the ideal $\left\langle\omega_{i}^{2}\right\rangle_{i \in \mathbb{N} \backslash\{0\}}$ is mapped to zero. So to write $\left\langle\omega_{i}^{2}\right\rangle_{i \in \mathbb{N} \backslash\{0\}}=\operatorname{ker} f^{*}$

Composing $f$ with the projection $p: B \mathcal{O} \rightarrow B U$, I obtain a constant map (the whole fibre is mapped to its basepoint) and therefore a trivial bundle $(p \circ f)^{*} \gamma\left(\mathbb{C}^{\infty}\right)$. This pullback of the complex universal bundle happens to be the complexification of $f^{*} \gamma\left(\mathbb{R}^{\infty}\right)$ :

$$
\begin{aligned}
& (p \circ f)^{*} \gamma\left(\mathbb{C}^{\infty}\right)=f^{*} p^{*} E U \times_{U} \mathbb{C}^{\infty}=f^{*} E \mathcal{O} \times_{U} \mathbb{C}^{\infty}=f^{*}\left(E \mathcal{O} \times_{\mathcal{O}} \mathbb{R}^{\infty}\right)^{\mathbb{C}} \\
& =f^{*} \gamma\left(\mathbb{R}^{\infty}\right)^{\mathbb{C}}=\left(f^{*} \gamma\left(\mathbb{R}^{\infty}\right)\right)^{\mathbb{C}} .
\end{aligned}
$$

So, $f^{*} \gamma\left(\mathbb{R}^{\infty}\right)$ admits a trivial complexification, and all of the transferred stable invariant classes $c$ must treat it like the trivial bundle $\varepsilon$ :

$c\left(f^{*} \gamma\left(\mathbb{R}^{\infty}\right)\right)=c(\varepsilon)$. A pullback of the trivial bundle is trivial too, so

$0=c\left(f^{*} \gamma\left(\mathbb{R}^{\infty}\right)\right)-c\left(f^{*} \varepsilon\right)=f^{*}\left(c\left(\gamma\left(\mathbb{R}^{\infty}\right)\right)-c(\varepsilon)\right)$ by naturality.

$\Rightarrow c\left(\gamma\left(\mathbb{R}^{\infty}\right)\right)-c(\varepsilon) \epsilon \operatorname{ker} f^{*}=$ * $\left\langle\omega_{i}^{2}\right\rangle_{i \in \mathbb{N} \backslash\{0\}}$.

Goal. I want to get a decomposition $c\left(\gamma\left(\mathbb{R}^{\infty}\right)\right)-c(\varepsilon)$

$$
\begin{aligned}
& =\sum_{j_{1}=1}^{m} \omega_{i_{j_{1}}}^{2} \cup \sum_{j_{2}=1}^{m_{j_{1}}} \omega_{i_{\left(j_{1}, j_{2}\right)}}^{2} \cup \sum \ldots \cup \sum_{j_{k}=1}^{m_{\left(j_{1}, \ldots, j_{k-1}\right)}} \omega_{i_{\left(j_{1}, \ldots, j_{k}\right)}}^{2} \cup r_{\left(j_{1}, \ldots, j_{k}\right)}\left(\gamma\left(\mathbb{R}^{\infty}\right)\right) \\
& +\sum_{j_{1}=1}^{m} \omega_{i_{j_{1}}}^{2} \cup r_{j_{1}}(\varepsilon)+\ldots+\sum_{j_{1}=1}^{m} \omega_{i_{j_{1}}}^{2} \cup \sum \ldots \cup \sum_{j_{k-1}=1}^{m_{\left(j_{1}, \ldots, j_{k-2}\right)}} \omega_{i_{\left(j_{1}, \ldots, j_{k-1}\right)}^{2}}^{2} \cup r_{\left(j_{1}, \ldots, j_{k-1}\right)}(\varepsilon)
\end{aligned}
$$

for some $m, m_{j_{1}}, \ldots, m_{\left(j_{1}, \ldots, j_{k-1}\right)} \in \mathbb{N} \cup\{0\}$, some $i_{j_{1}}, \ldots, i_{\left(j_{1}, \ldots, j_{k}\right)} \in \mathbb{N} \backslash\{0\}$, some $r_{\left(j_{1}, \ldots, j_{k}\right)}\left(\gamma\left(\mathbb{R}^{\infty}\right)\right) \in H^{*}\left(B \mathcal{O}, \mathbb{Z}_{2}\right)$,

and some coefficients $r_{j_{1}}(\varepsilon), \ldots, r_{\left(j_{1}, \ldots, j_{k-1}\right)}(\varepsilon) \in\{0,1\}$,

in a way that $\forall \vec{j}:=\left(j_{1}, \ldots, j_{k}\right): \quad 2 \sum_{p \in I(\vec{j})} p>\operatorname{deg} c$,

where $I(\vec{j}):=\left\{i_{j_{1}}, \ldots, i_{\left(j_{1}, \ldots, j_{k}\right)}\right\}$.

Being arrived at this goal and knowing that the degree must be the same on both sides of the equation, the sum over all terms containing a factor $\bigcup_{p \in I(\vec{j})} \omega_{p}^{2}$ of too high degree $2 \sum_{p \in I(\vec{j})} p$, for any $\vec{j}$, must vanish. 
So, a polynomial $c\left(\gamma\left(\mathbb{R}^{\infty}\right)\right)$ in some squares $\omega_{p}^{2}, p \in \mathbb{N} \cup\{0\}$ will remain ${ }^{3}$ $c\left(\gamma\left(\mathbb{R}^{\infty}\right)\right)=c(\varepsilon)+\sum_{j_{1}=1}^{m} \omega_{i_{j_{1}}}^{2} \cup r_{j_{1}}(\varepsilon)+\ldots+\ldots$
$+\sum_{j_{1}=1}^{m} \omega_{i_{j_{1}}}^{2} \cup \sum \ldots \cup \sum_{j_{k-1}=1}^{m_{\left(j_{1}, \ldots, j_{k-2}\right)}} \omega_{i_{\left(j_{1}, \ldots, j_{k-1}\right)}^{2}}^{2} \cup r_{\left(j_{1}, \ldots, j_{k-1}\right)}(\varepsilon)$.

Before beginning, I should introduce two notions just to make the proof more readable:

Definition. An index vector $\vec{j}$ "appears" in a given decomposition of $c\left(\gamma\left(\mathbb{R}^{\infty}\right)\right)-c(\varepsilon)$, if there is a summand $r_{\vec{j}}\left(\gamma\left(\mathbb{R}^{\infty}\right)\right) \cup \underset{p \in I(\vec{j})}{\bigcup} \omega_{p}^{2}$ visible in this decomposition, and if $2 \sum_{p \in I(\vec{j})} p \leq \operatorname{deg} c$.

$\underline{\text { Remark. }}$ The terms $r_{\vec{j}}\left(\gamma\left(\mathbb{R}^{\infty}\right)\right) \cup \bigcup_{p \in I(\vec{j})} \omega_{p}^{2}$ with $2 \sum_{p \in I(\vec{j})} p>\operatorname{deg} c$ must

vanish in any decomposition of $c\left(\gamma\left(\mathbb{R}^{\infty}\right)\right)-c(\varepsilon)$. That's why I don't let them contribute in the last definition.

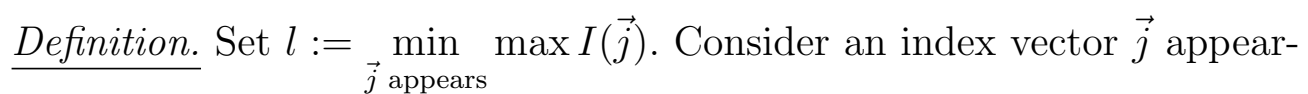
ing in a given decomposition of $c\left(\gamma\left(\mathbb{R}^{\infty}\right)\right)-c(\varepsilon)$. If $\max I(\vec{j})=l$, then call $r_{\vec{j}}\left(\gamma\left(\mathbb{R}^{\infty}\right)\right)-r_{\vec{j}}(\varepsilon)$ a "low situated rest term".

As seen so far, $c\left(\gamma\left(\mathbb{R}^{\infty}\right)\right)-c(\varepsilon) \epsilon \operatorname{ker} f^{*}=\left\langle\omega_{i}^{2}\right\rangle_{i \in \mathbb{N} \backslash\{0\}}$, so there is a decomposition

$$
c\left(\gamma\left(\mathbb{R}^{\infty}\right)\right)-c(\varepsilon)=\sum_{j_{1}=1}^{m} \omega_{i_{j_{1}}}^{2} \cup r_{j_{1}}\left(\gamma\left(\mathbb{R}^{\infty}\right)\right),
$$

for some $m \in \mathbb{N} \cup\{0\}$, some $i_{j_{1}} \in \mathbb{N} \backslash\{0\}$, some $r_{j_{1}}\left(\gamma\left(\mathbb{R}^{\infty}\right)\right) \in H^{*}\left(B \mathcal{O}, \mathbb{Z}_{2}\right)$. I will show that there's a low situated rest term $r_{j_{1}}\left(\gamma\left(\mathbb{R}^{\infty}\right)\right)-r_{j_{1}}(\varepsilon)$ in this decomposition that lies in ker $f^{*}$. Then, that low situated rest term admits a decomposition as a linear combination of squares $\omega_{i_{\left(j_{1}, j_{2}\right)}}^{2}$ with coefficients $r_{\left(j_{1}, j_{2}\right)}\left(\gamma\left(\mathbb{R}^{\infty}\right)\right) \in H^{*}\left(B \mathcal{O}, \mathbb{Z}_{2}\right)$, leading to a new decomposition of

$c\left(\gamma\left(\mathbb{R}^{\infty}\right)\right)-c(\varepsilon)$. So, inductively, I will replace a low situated rest term in any given decomposition of $c\left(\gamma\left(\mathbb{R}^{\infty}\right)\right)-c(\varepsilon)$ by a linear combination whose

\footnotetext{
${ }^{3}$ The classes $c(\varepsilon), r_{\vec{j}}(\varepsilon)$ of the trivial bundle $\varepsilon$ are just coefficients in $H^{0}\left(B \mathcal{O}, \mathbb{Z}_{2}\right)=\{0,1\}$.
} 
coefficients are rest terms with longer index vectors. That's why after a finite number of these steps, the index vectors $\vec{j}$ won't "appear" no more, because the sums $2 \sum_{p \in I(\vec{j})} p$ will exceed the degree of $c$. That's the moment when all low situated rest terms are eliminated and the decomposition described in my goal is achieved.

To do all this, I first need to introduce a procedure that shall be called:

"Cutting the equation $c(F \oplus G)=c(G)$ at the dimension l". Define the bundles

$$
\begin{aligned}
F & :=p r_{1}^{*} f^{*} \gamma\left(\mathbb{R}^{\infty}\right) \longrightarrow U / \mathcal{O} \times B \mathcal{O} \text { and } \\
G & :=p r_{2}^{*} \gamma\left(\mathbb{R}^{\infty}\right) \longrightarrow U / \mathcal{O} \times B \mathcal{O},
\end{aligned}
$$

where $p r_{i}$ shall be the projection on the $i$-th factor of the base space $U / \mathcal{O} \times B \mathcal{O}$. Let $l \in \mathbb{N}$.

Consider the map $\left(i d, e m b_{l}\right):\left(U / \mathcal{O} \times B \mathcal{O}_{l}\right) \hookrightarrow(U / \mathcal{O} \times B \mathcal{O})$,

where $e m b_{l}: B \mathcal{O}_{l} \hookrightarrow B \mathcal{O}$ shall be the natural embedding, recalling that $B \mathcal{O}$ is the limes space over all $B \mathcal{O}_{l}, l \in \mathbb{N}$. Then the bundle $G_{l}:=\left(i d, e m b_{l}\right)^{*} G$ admits Stiefel-Whitney classes that are in bijective correspondence with those of the $l$-dimensional universal bundle $\gamma_{l}\left(\mathbb{R}^{\infty}\right) \rightarrow B \mathcal{O}_{l}$.

(To be precise, $G_{l} \cong p r_{B \mathcal{O}_{l}}{ }^{*} \gamma_{l}\left(\mathbb{R}^{\infty}\right)$, the situation being

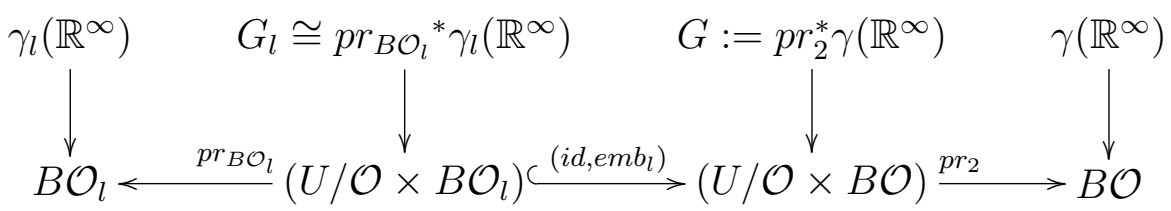

Especially, $w_{p}\left(G_{l}\right)$ vanishes for $p>l$.

The bundle $F$ inherits from $f^{*} \gamma\left(\mathbb{R}^{\infty}\right)$ the property to admit a trivial complexification.

Therefore, the "transferred stable invariance" of $c$ applies:

$$
c(F \oplus G)=c(G) .
$$

Thus, applying the induced cohomology map $\left(i d, e m b_{l}\right)^{*}$ gives

$$
\left(i d, e m b_{l}\right)^{*} c(F \oplus G)=\left(i d, e m b_{l}\right)^{*} c(G)
$$




$$
\begin{gathered}
\Leftrightarrow c\left(i d^{*} F \oplus e m b_{l}^{*} G\right)=c\left(e m b_{l}^{*} G\right) \\
\Leftrightarrow c\left(F \oplus G_{l}\right)=c\left(G_{l}\right) .
\end{gathered}
$$

By the universality of $\gamma\left(\mathbb{R}^{\infty}\right)$, and the naturality of all characteristic classes towards the classifying maps of $G_{l}$ and $F \oplus G_{l}$, any given decomposition

$$
\begin{aligned}
& c\left(\gamma\left(\mathbb{R}^{\infty}\right)\right)-c(\varepsilon)=\sum_{\vec{j}} r_{\vec{j}}\left(\gamma\left(\mathbb{R}^{\infty}\right)\right) \bigcup_{p \in I(\vec{j})} \omega_{p}^{2} \text { gives analogous decompositions } \\
& c\left(G_{l}\right)-c(\varepsilon)=\sum_{\vec{j}} r_{\vec{j}}\left(G_{l}\right) \bigcup_{p \in I(\vec{j})} w_{p}^{2}\left(G_{l}\right) \quad \text { and } \\
& c\left(F \oplus G_{l}\right)-c(\varepsilon)=\sum_{\vec{j}} r_{\vec{j}}\left(F \oplus G_{l}\right) \bigcup_{p \in I(\vec{j})} w_{p}^{2}\left(F \oplus G_{l}\right) .
\end{aligned}
$$

Assertion 1 gives the "transferred stable invariance" of $w_{p}^{2}$, making it invariant under adding the bundle $F$, whose complexification is trivial :

$w_{p}^{2}\left(F \oplus G_{l}\right)=w_{p}^{2}\left(G_{l}\right)$.

Thus, the equation $c\left(F \oplus G_{l}\right)=c\left(G_{l}\right)$ can be rewritten as:

$$
\sum_{\vec{j}} r_{\vec{j}}\left(F \oplus G_{l}\right) \bigcup_{p \in I(\vec{j})} w_{p}^{2}\left(G_{l}\right)=\sum_{\vec{j}} r_{\vec{j}}\left(G_{l}\right) \bigcup_{p \in I(\vec{j})} w_{p}^{2}\left(G_{l}\right)
$$

where all summands containing a factor $w_{p}\left(G_{l}\right)$ with $p>l$ vanish:

$$
\Leftrightarrow \sum_{\vec{j}}^{\max I(\vec{j}) \leq l} r_{\vec{j}}\left(F \oplus G_{l}\right) \bigcup_{p \in I(\vec{j})} w_{p}^{2}\left(G_{l}\right)=\sum_{\vec{j}}^{\max I(\vec{j}) \leq l} r_{\vec{j}}\left(G_{l}\right) \bigcup_{p \in I(\vec{j})} w_{p}^{2}\left(G_{l}\right)
$$

For not to exceed the degree of $c$, also all terms with

$2 \sum_{p \in I(\vec{j})} p>\operatorname{deg} c$ must vanish:

$$
\Rightarrow \sum_{\vec{j} \text { appears }}^{\max I(\vec{j}) \leq l} r_{\vec{j}}\left(F \oplus G_{l}\right) \bigcup_{p \in I(\vec{j})} w_{p}^{2}\left(G_{l}\right)=\sum_{\vec{j} \text { appears }}^{\max I(\vec{j}) \leq l} r_{\vec{j}}\left(G_{l}\right) \bigcup_{p \in I(\vec{j})} w_{p}^{2}\left(G_{l}\right)
$$

So, it's this last expression that I'll call "the equation $c(F \oplus G)=c(G)$ cut at the dimension $l$ ". 
$\underline{\text { Induction over the index vector pointing at a low situated rest term }}$

Induction's beginning. Recall $c\left(\gamma\left(\mathbb{R}^{\infty}\right)\right)-c(\varepsilon)=\sum_{j_{1}=1}^{m} \omega_{i_{j_{1}}}^{2} \cup r_{j_{1}}\left(\gamma\left(\mathbb{R}^{\infty}\right)\right)$.

Rename $i_{1}, \ldots, i_{m}$ such that $i_{1}<i_{2}<\ldots<i_{m}$.

Cut the equation $c(F \oplus G)=c(G)$ at $i_{1}$, and get

$$
\sum_{j_{1}}^{i_{j_{1}} \leq i_{1}} r_{j_{1}}\left(F \oplus G_{i_{1}}\right) \cup w_{i_{j_{1}}}^{2}\left(G_{i_{1}}\right)=\sum_{j_{1} \text { appears }}^{i_{j_{1}} \leq i_{1}} r_{j_{1}}\left(G_{i_{1}}\right) \cup w_{i_{j_{1}}}^{2}\left(G_{i_{1}}\right) \text {. }
$$

As $i_{1}<i_{2}<\ldots<i_{m}$, this is just $r_{1}\left(F \oplus G_{i_{1}}\right) \cup w_{i_{1}}^{2}\left(G_{i_{1}}\right)=r_{1}\left(G_{i_{1}}\right) \cup w_{i_{1}}^{2}\left(G_{i_{1}}\right)$.

Injectivity of the multiplication map $\cup w_{i_{1}}^{2}\left(G_{i_{1}}\right)$ in $H^{*}\left(U / \mathcal{O} \times B \mathcal{O}_{i_{1}}, \mathbb{Z}_{2}\right)$ then holds $r_{1}\left(F \oplus G_{i_{1}}\right)=r_{1}\left(G_{i_{1}}\right)$.

Then pull this back with $(i d \times$ const $): U / \mathcal{O} \rightarrow\left(U / \mathcal{O} \times B \mathcal{O}_{i_{1}}\right)$, (where the map const takes just one, arbitrary, value), to get

$r_{1}\left(f^{*} \gamma\left(\mathbb{R}^{\infty}\right) \oplus \varepsilon\right)=r_{1}(\varepsilon)$.

Due to the stability of the Stiefel-Whitney classes [Hatcher, page 81], that's

$r_{1}\left(f^{*} \gamma\left(\mathbb{R}^{\infty}\right)\right)=r_{1}(\varepsilon)$.

Using naturality of characteristic classes towards pullbacks, this gives

$f^{*}\left(r_{1}\left(\gamma\left(\mathbb{R}^{\infty}\right)\right)-r_{1}(\varepsilon)\right)=0$. Or, $r_{1}\left(\gamma\left(\mathbb{R}^{\infty}\right)\right)-r_{1}(\varepsilon)$ lies in ker $f^{*}$.

So, I can replace it with a linear combination of quadratic terms, providing a new decomposition, $\quad c\left(\gamma\left(\mathbb{R}^{\infty}\right)\right)-c(\varepsilon)$

$$
=\omega_{i_{1}}^{2} \cup \sum_{j_{2}=1}^{m_{1}} \omega_{i_{\left(1, j_{2}\right)}}^{2} \cup r_{\left(1, j_{1}\right)}\left(\gamma\left(\mathbb{R}^{\infty}\right)\right)+\omega_{i_{1}}^{2} \cup r_{1}(\varepsilon)+\sum_{j_{1}=2}^{m} \omega_{i_{j_{1}}}^{2} \cup r_{j_{1}}\left(\gamma\left(\mathbb{R}^{\infty}\right)\right) .
$$

\section{Induction's prerequisite.}

Consider a given decomposition

$$
\begin{aligned}
& c\left(\gamma\left(\mathbb{R}^{\infty}\right)\right)-c(\varepsilon)=\sum_{\vec{j}} r_{\vec{j}}\left(\gamma\left(\mathbb{R}^{\infty}\right)\right) \bigcup_{p \in I(\vec{j})} \omega_{p}^{2} \\
& +\sum_{j_{1}=1}^{m} \omega_{i_{j_{1}}}^{2} \cup r_{j_{1}}(\varepsilon)+\ldots+\sum_{j_{1}=1}^{m} \omega_{i_{j_{1}}}^{2} \cup \sum \ldots \cup \sum_{j_{k-1}=1}^{m_{\left(j_{1}, \ldots, j_{k-2}\right)}} \omega_{i_{\left(j_{1}, \ldots, j_{k-1}\right)}}^{2} \cup r_{\left(j_{1}, \ldots, j_{k-1}\right)}(\varepsilon) .
\end{aligned}
$$

Induction's claim. There's a low situated rest term in this given decomposition that lies in $\operatorname{ker} f^{*}$. 
Induction's step. Cut the equation $c(F \oplus G)=c(G)$ at the dimension

$$
l:=\underset{\vec{j} \text { appears }}{\min } \max I(\vec{j})
$$

Then the remaining terms of $c\left(G_{l}\right)-c(\varepsilon)$ do all have the common factor $w_{l}^{2}\left(G_{l}\right)$. This is no zero divisor in $H^{*}\left(U / \mathcal{O} \times B \mathcal{O}_{l}, \mathbb{Z}_{2}\right)$ and further its multiplication map $\cup w_{l}^{2}\left(G_{l}\right)$ is injective. Now, in $c\left(F \oplus G_{l}\right)=c\left(G_{l}\right)$

$$
\Rightarrow \sum_{\vec{j} \text { appears }}^{\max I(\vec{j}) \leq l} r_{\vec{j}}\left(F \oplus G_{l}\right) \bigcup_{p \in I(\vec{j})} w_{p}^{2}\left(G_{l}\right)=\sum_{\vec{j} \text { appears }}^{\max I(\vec{j}) \leq l} r_{\vec{j}}\left(G_{l}\right) \bigcup_{p \in I(\vec{j})} w_{p}^{2}\left(G_{l}\right),
$$

this injectivity delivers

$$
\Rightarrow \sum_{\vec{j} \text { appears }}^{\max I(\vec{j}) \leq l} r_{\vec{j}}\left(F \oplus G_{l}\right) \bigcup_{p \in I(\vec{j}) \backslash\{l\}} w_{p}^{2}\left(G_{l}\right)=\sum_{\vec{j} \text { appears }}^{\max I(\vec{j}) \leq l} r_{\vec{j}}\left(G_{l}\right) \bigcup_{p \in I(\vec{j}) \backslash\{l\}} w_{p}^{2}\left(G_{l}\right) .
$$

$\diamond$ If there is just one low situated rest term $r_{\vec{j}}\left(\gamma\left(\mathbb{R}^{\infty}\right)\right)-r_{\vec{j}}(\varepsilon)$, then use the injectivity of the multiplication map $\cup \bigcup_{p \in I(\vec{j}) \backslash\{l\}} w_{p}^{2}\left(G_{l}\right)$

in $H^{*}\left(U / \mathcal{O} \times B \mathcal{O}_{l}, \mathbb{Z}_{2}\right)$ to obtain $r_{\vec{j}}\left(F \oplus G_{l}\right)=r_{\vec{j}}\left(G_{l}\right)$. Then pull this back with

$$
\begin{aligned}
& (i d \times \text { const }): U / \mathcal{O} \rightarrow\left(U / \mathcal{O} \times B \mathcal{O}_{l}\right) \quad \text { to get } \quad r_{\vec{j}}\left(f^{*} \gamma\left(\mathbb{R}^{\infty}\right) \oplus \varepsilon\right)=r_{\vec{j}}(\varepsilon) \\
& \Rightarrow r_{\vec{j}}\left(f^{*} \gamma\left(\mathbb{R}^{\infty}\right)\right)=r_{\vec{j}}(\varepsilon) \text {. Using naturality, this means } \\
& f^{*}\left(r_{\vec{j}}\left(\gamma\left(\mathbb{R}^{\infty}\right)\right)-r_{\vec{j}}(\varepsilon)\right)=0 . \\
& \Rightarrow \text { The low situated rest term } r_{\vec{j}}\left(\gamma\left(\mathbb{R}^{\infty}\right)\right)-r_{\vec{j}}(\varepsilon) \text { lies in ker } f^{*} .
\end{aligned}
$$


$\diamond$ Else cut the remaining equation again at the dimension

$$
l^{\prime}:=\underset{\vec{j} \text { appears }}{\max I(\vec{j})=l} \max (I(\vec{j}) \backslash\{l\}), \quad \text { such as to obtain }
$$

$\sum_{\vec{j} \text { appears }}^{\max (I(\vec{j}) \backslash\{l\}) \leq l^{\prime}} r_{\vec{j}}\left(F \oplus G_{l^{\prime}}\right) \bigcup_{p \in(I(\vec{j}) \backslash\{l\})} w_{p}^{2}\left(G_{l^{\prime}}\right)=\sum_{\vec{j} \text { appears }}^{\max (I(\vec{j}) \backslash\{l\}) \leq l^{\prime}} r_{\vec{j}}\left(G_{l^{\prime}}\right) \bigcup_{p \in(I(\vec{j}) \backslash\{l\})} w_{p}^{2}\left(G_{l^{\prime}}\right)$.

Now proceed analogously with the choice marked with the " $\diamond "$ signs, and after finitely many steps, find a low situated rest term in ker $f^{*}$.

This low situated rest term can be replaced by a linear combination of squares, holding a new decomposition of $c\left(\gamma\left(\mathbb{R}^{\infty}\right)\right)-c(\varepsilon)$.

This completes the induction.

By the universality of $\gamma\left(\mathbb{R}^{\infty}\right)$,

$$
\begin{aligned}
& c=c(\varepsilon)+\sum_{j_{1}=1}^{m} w_{i_{j_{1}}}^{2} \cup r_{j_{1}}(\varepsilon)+\ldots+\ldots \\
& +\sum_{j_{1}=1}^{m} w_{i_{j_{1}}}^{2} \cup \sum \ldots \cup \sum_{j_{k-1}=1}^{m_{\left(j_{1}, \ldots, j_{k-2}\right)}} w_{i_{\left(j_{1}, \ldots, j_{k-1}\right)}^{2}}^{2} \cup r_{\left(j_{1}, \ldots, j_{k-1}\right)}(\varepsilon) .
\end{aligned}
$$

As $c(\varepsilon), r_{j_{1}}(\varepsilon), \ldots, r_{\left(j_{1}, \ldots, j_{k-1}\right)}(\varepsilon) \in\left\{0,1=w_{0}=w_{0}^{2}\right\}$ classes.

$c$ is in the sub-ring $\mathbb{Z}_{2}\left[w_{i}^{2}\right]_{i \in \mathbb{N} \cup\{0\}}$ of the polynomial ring of Stiefel-Whitney

So, Assertion 2 is proved. 


\subsection{Using integral coefficients}

I will lean on the obtained results for $\mathbb{Z}_{2}$-coefficients and use the

$\bmod 2$-reduction homomorphism $\rho: H^{*}(B \mathcal{O}, \mathbb{Z}) \rightarrow H^{*}\left(B \mathcal{O}, \mathbb{Z}_{2}\right)$

to pass over to answering the same questions in $\mathbb{Z}$-coefficients.

The cohomology ring of $B \mathcal{O}$ with $\mathbb{Z}$-coefficients is known with all relations between its generators since [Brown] and can be obtained as follows:

Define the set of generators of $H^{*}\left(B \mathcal{O}_{n}, \mathbb{Z}\right)$ as in [Feshbach, page 511, Definition 1]:

It consists of the Pontrjagin classes $p_{i}$ of the universal bundle over $B \mathcal{O}_{n}$, and classes $V_{I}$ with $I$ ranging over all finite nonempty subsets of

$$
\left\{\frac{1}{2}\right\} \cup\left\{k \in \mathbb{Z} \mid 0<k<\frac{n+1}{2}\right\}
$$

with the proviso that $I$ does not contain both $\frac{1}{2}$ and $\frac{n}{2}$, for $n>1$.

According to [Feshbach, page 512, Theorem 2], $H^{*}\left(B \mathcal{O}_{n}, \mathbb{Z}\right)$ is for all $n \leq \infty$ isomorphic to the polynomial ring over $\mathbb{Z}$ generated by the above specified elements modulo the ideal generated by the following six types of relations.

In all relations except the first, the cardinality of $I$ is less than or equal to that of $J$ and greater than one. (Most of the restrictions on $I$ and $J$ are to avoid repeating relations). By convention, $p_{\frac{1}{2}}$ where it occurs means $V_{\left\{\frac{1}{2}\right\}}$. Also, if $\left\{\frac{n}{2}, \frac{1}{2}\right\} \subset I \cup J$, then $V_{I \cup J}$ shall mean $V_{\left\{\frac{n}{2}\right\}} V_{(I \cup J) \backslash\left\{\frac{n}{2}, \frac{1}{2}\right\}}$.

1) $2 V_{I}=0$.

2) $V_{I} V_{J}+V_{I \cup J} V_{I \cap J}+V_{I \backslash J} V_{J \backslash I} \prod_{i \in I \cap J} p_{i}=0 \quad($ for $I \cap J \neq \emptyset, I \nsubseteq J)$.

3) $V_{I} V_{J}+\sum_{i \in I} V_{\{i\}} V_{(J \backslash I) \cup\{i\}} \prod_{j \in I \backslash\{i\}} p_{j}=0 \quad($ for $I \subset J)$.

4) $V_{I} V_{J}+\sum_{i \in I} V_{\{i\}} V_{(I \cup J) \backslash\{i\}}=0 \quad$ (for $I \cap J=\emptyset$; if $I$ and $J$ have the same cardinality, then the smallest element of $I$ is less than that of $J$ ).

5) $\sum_{i \in I} V_{\{i\}} V_{I \backslash\{i\}}=0$.

6) $V_{\left\{\frac{1}{2}\right\}} p_{\frac{n}{2}}+V_{\left\{\frac{n}{2}\right\}}^{2}=0$, if $\mathrm{n}$ is even. 
Then $\rho\left(V_{I}\right)=S q^{1}\left(\bigcup_{i \in I} w_{2 i}\right)$,

with the Steenrod squaring operation $S q^{1}$.

Assertion 3. The basic requirement holds for $V_{I}^{2}$, with $I$ arbitrary.

\section{Lemma.}

$$
\begin{aligned}
& \rho\left(V_{I}^{2}(\xi)\right)=\sum_{i \in I \cap\left\{\frac{1}{2}\right\}} w_{1}^{2}(\xi \oplus \xi) \cup \bigcup_{j \in I \backslash\{i\}} w_{4 j}(\xi \oplus \xi) \\
& +\sum_{i \in I \backslash\left\{\frac{1}{2}\right\}}\left(w_{4 i+2}(\xi \oplus \xi)+w_{2}(\xi \oplus \xi) \cup w_{4 i}(\xi \oplus \xi)\right) \cup \bigcup_{j \in I \backslash\{i\}} w_{4 j}(\xi \oplus \xi),
\end{aligned}
$$

for any real bundle $\xi$.

Proof. Apply the reduction homomorphism:

$$
\begin{aligned}
& \rho\left[V_{I}^{2}(\xi)\right]=\left(\rho\left[V_{I}(\xi)\right]\right)^{2}=\left(S q^{1}\left[\bigcup_{i \in I} w_{2 i}(\xi)\right]\right)^{2}=\left(\sum_{i \in I} S q^{1}\left[w_{2 i}(\xi)\right] \cup \bigcup_{j \in I \backslash\{i\}} w_{2 j}(\xi)\right)^{2} \\
& =\left[\sum_{i \in I \cap\left\{\frac{1}{2}\right\}} w_{1}^{2}(\xi) \cup \bigcup_{j \in I \backslash\{i\}} w_{2 j}(\xi)\right. \\
& \left.+\sum_{i \in I \backslash\left\{\frac{1}{2}\right\}}\left(w_{2 i+1}(\xi)+w_{1}(\xi) \cup w_{2 i}(\xi)\right) \cup \bigcup_{j \in I \backslash\{i\}} w_{2 j}(\xi)\right]^{2} .
\end{aligned}
$$

As $2=0$ in $H^{*}\left(B \mathcal{O}, \mathbb{Z}_{2}\right)$, this equals

$$
\begin{aligned}
& =\sum_{i \in I \cap\left\{\frac{1}{2}\right\}} w_{1}^{4}(\xi) \cup \bigcup_{j \in I \backslash\{i\}} w_{2 j}^{2}(\xi) \\
& +\sum_{i \in I \backslash\left\{\frac{1}{2}\right\}}\left(w_{2 i+1}^{2}(\xi)+w_{1}^{2}(\xi) \cup w_{2 i}^{2}(\xi)\right) \cup \bigcup_{j \in I \backslash\{i\}} w_{2 j}^{2}(\xi) .
\end{aligned}
$$

Using the Whitney sum axiom and symmetry,

$w_{4 i}(\xi \oplus \xi)=\sum_{k=1}^{4 i} w_{k}(\xi) w_{4 i-k}(\xi)=w_{2 i}^{2}(\xi)$. Hence, the above term equals

$$
\begin{aligned}
& \sum_{i \in I \cap\left\{\frac{1}{2}\right\}} w_{1}^{2}(\xi \oplus \xi) \cup \bigcup_{j \in I \backslash\{i\}} w_{4 j}(\xi \oplus \xi) \\
+ & \sum_{i \in I \backslash\left\{\frac{1}{2}\right\}}\left(w_{4 i+2}(\xi \oplus \xi)+w_{2}(\xi \oplus \xi) \cup w_{4 i}(\xi \oplus \xi)\right) \cup \bigcup_{j \in I \backslash\{i\}} w_{4 j}(\xi \oplus \xi)
\end{aligned}
$$


Proof of assertion 3.

Let $F \rightarrow B, G \rightarrow B$ be real bundles with $F^{\mathbb{C}} \cong G^{\mathbb{C}}$. Forgetting the complex structure, that's $F \oplus F \cong G \oplus G$. By naturality of the StiefelWhitney classes,

$$
\begin{aligned}
& \sum_{i \in I \cap\left\{\frac{1}{2}\right\}} w_{1}^{2}(F \oplus F) \cup \bigcup_{j \in I \backslash\{i\}} w_{4 j}(F \oplus F) \\
+ & \sum_{i \in I \backslash\left\{\frac{1}{2}\right\}}\left(w_{4 i+2}(F \oplus F)+w_{2}(F \oplus F) \cup w_{4 i}(F \oplus F)\right) \cup \bigcup_{j \in I \backslash\{i\}} w_{4 j}(F \oplus F) \\
= & \sum_{i \in I \cap\left\{\frac{1}{2}\right\}} w_{1}^{2}(G \oplus G) \cup \bigcup_{j \in I \backslash\{i\}} w_{4 j}(G \oplus G) \\
+ & \sum_{i \in I \backslash\left\{\frac{1}{2}\right\}}\left(w_{4 i+2}(G \oplus G)+w_{2}(G \oplus G) \cup w_{4 i}(G \oplus G)\right) \cup \bigcup_{j \in I \backslash\{i\}} w_{4 j}(G \oplus G)
\end{aligned}
$$

for any finite nonempty index set $I \subset\left(\left\{\frac{1}{2}\right\} \cup \mathbb{N} \backslash\{0\}\right)$. Applying the lemma, this means $\rho\left(V_{I}^{2}(F)\right)=\rho\left(V_{I}^{2}(G)\right)$.

As $V_{I}^{2}$ is in the torsion of $H^{*}(B \mathcal{O}, \mathbb{Z})$, restricted on which $\rho$ is injective [Feshbach, page 513, four lines above "Case 1"], this proves the assertion: $V_{I}^{2}(F)=V_{I}^{2}(G)$.

In the same way, it has to be dealt with $V_{\left\{\frac{1}{2}\right\}}$ to see that it meets the basic requirement, as $\rho\left(V_{\left\{\frac{1}{2}\right\}}\right)=S q^{1}\left(w_{1}\right)=w_{1}^{2}$.

Also the Pontrjagin classes $p_{i}$ fulfill the basic requirement:

$$
p_{i}(F)=(-1)^{i} c_{2 i}\left(F^{\mathbb{C}}\right)=(-1)^{i} c_{2 i}\left(G^{\mathbb{C}}\right)=p_{i}(G) .
$$

\section{Corollary from assertion 2}

Let $C \in H^{*}(B \mathcal{O}, \mathbb{Z})$ fulfill the basic requirement. Then $\rho(C) \in \mathbb{Z}_{2}\left[w_{i}^{2}\right]_{i \in \mathbb{N} \cup\{0\}}$. Proof.

Let $F \rightarrow B, G \rightarrow B$ be real bundles with $F^{\mathbb{C}} \cong G^{\mathbb{C}}$. The reduction $\rho(C) \in H^{*}\left(B \mathcal{O}, \mathbb{Z}_{2}\right)$ also satisfies the basic requirement:

$$
\rho(C)(F)=\rho(C(F))=\rho(C(G))=\rho(C)(G) .
$$

Thus claim 1 on page 34 says it's transferred stable invariant. Assertion 2 now gives the result. 


\section{Estimation}

The sub-ring $R$ of $H^{*}(B \mathcal{O}, \mathbb{Z})$ generated by $p_{i}$ with $i \in \mathbb{N}, V_{J}^{2}$ where $\emptyset \neq J \subset\left(\left\{\frac{1}{2}\right\} \cup \mathbb{N} \backslash\{0\}\right)$, and $V_{\left\{\frac{1}{2}\right\}}$ should contain all well-defined integral cohomology classes of "real bone" bundles.

This estimation is not needed for the following conclusion. So I ought to give only some hand-waving arguments for it.

Take a class $C \in H^{*}(B \mathcal{O}, \mathbb{Z}) \backslash R$ which fulfills the basic requirement.

Then, $\rho(C) \in \mathbb{Z}_{2}\left[w_{i}^{2}\right]_{i \in \mathbb{N} \cup\{0\}}$ according to the corollary from assertion 2 .

Therefore $\rho(C)$ is a polynomial of squares $\sum \bigcup w_{j}^{2}$.

On the other hand $H^{*}(B \mathcal{O}, \mathbb{Z})=\mathbb{Z}\left[p_{i}\right]_{i \in \mathbb{N}} \oplus 2$-Torsion with $\mathbb{Z}\left[p_{i}\right]_{i \in \mathbb{N}}$,

$\left\{V_{J}^{2}\right\}_{\emptyset \neq J \subset\left(\left\{\frac{1}{2}\right\} \cup \mathbb{N} \backslash\{0\}\right)},\left\{V_{\left\{\frac{1}{2}\right\}}\right\}$ subsets of $R$; thus there must be a torsion generator $V_{I}, I \neq\left\{\frac{1}{2}\right\}$, as a factor in a summand $C^{(s)}$ of $C$, appearing in an odd potence $V_{I}^{2 k+1}$.

Thus the reduction $\rho\left(C^{(s)}\right)$ admits the non-quadratic term

$$
\rho\left(V_{I}\right)=\sum_{i \in I \cap\left\{\frac{1}{2}\right\}} w_{1}^{2} \cup \bigcup_{j \in I \backslash\{i\}} w_{2 j}+\sum_{i \in I \backslash\left\{\frac{1}{2}\right\}}\left(w_{2 i+1}+w_{1} \cup w_{2 i}\right) \cup \bigcup_{j \in I \backslash\{i\}} w_{2 j}
$$

as a factor. And as $2=0$ in $H^{*}\left(B \mathcal{O}, \mathbb{Z}_{2}\right), \sum \bigcup w_{j}^{2}$ equals $\left(\sum \bigcup w_{j}\right)^{2}$. Hence for $\rho\left(V_{I}\right)$ to be a factor in a polynomial of squares, it needs to be multiplied exactly with itself, and not only with the remainder $\rho\left(V_{I}^{2 k}\right)=$

$$
\left(\left(\rho V_{I}\right)^{2}\right)^{k}=\left(\sum_{i \in I \cap\left\{\frac{1}{2}\right\}} w_{1}^{4} \cup \bigcup_{j \in I \backslash\{i\}} w_{2 j}^{2}+\sum_{i \in I \backslash\left\{\frac{1}{2}\right\}}\left(w_{2 i+1}^{2}+w_{1}^{2} \cup w_{2 i}^{2}\right) \cup \bigcup_{j \in I \backslash\{i\}} w_{2 j}^{2}\right)^{k}
$$

or other factors of $\rho\left(C^{(s)}\right)$.

Other summands of $\rho(C)$ won't cancel out $\rho\left(C^{(s)}\right), \rho$ being injective on the torsion element:5.

That's why I think that $C$ can't fulfill the basic requirement.

\subsection{Conclusion}

In $\mathbb{Z}_{2}$-coefficients, assertion 2 yields that every well-defined characteristic class of the "real bone" bundles $\xi$ of a complex vector bundle $\xi^{\mathbb{C}}$ is a polynomial in the squares of Stiefel-Whitney classes

$\sum \bigcup w_{i}^{2}(\xi)=\sum \bigcup w_{2 i}(\xi \oplus \xi) \equiv \sum \bigcup c_{i}\left(\xi^{\mathbb{C}}\right) \bmod 2$, and thus determined by the Chern classes $c_{i}\left(\xi^{\mathbb{C}}\right)$.

\footnotetext{
${ }^{4}$ [Feshbach, page 513, eight lines above "Case 1"]

5 [Feshbach, page 513, four lines above "Case 1"]
} 
The following assertion states the same in $\mathbb{Z}$-coefficients.

\section{Assertion 4}

Let $C \in H^{*}(B \mathcal{O}, \mathbb{Z})$ be a well-defined characteristic class of "real bone bundles".

Then for any bundle $\xi, C(\xi)$ is completely determined by some Chern classes $c_{k}\left(\xi^{\mathbb{C}}\right), k \in \mathbb{N}$.

Proof.

[Feshbach, page 513, eight lines above "Case 1"] tells that

$H^{*}(B \mathcal{O}, \mathbb{Z})=\mathbb{Z}\left[p_{i}\right]_{i \in \mathbb{N}} \oplus$ 2-Torsion.

$\Rightarrow C=\sum \bigcup p_{i}+T$ with some torsion element $T \in H^{*}(B \mathcal{O}, \mathbb{Z})$

So for every real bundle $\xi$,

$$
\begin{aligned}
& \rho(C)(\xi)=\sum \rho\left(\bigcup p_{i}(\xi)\right)+\rho(T)(\xi) . \\
& \Rightarrow{ }^{6} \rho(C)(\xi)=\sum \bigcup \rho\left((-1)^{i} c_{2 i}\left(\xi^{\mathbb{C}}\right)\right)+\rho(T)(\xi) . \\
& \Rightarrow]^{7} \rho(C)(\xi)=\sum \bigcup w_{4 i}(\xi \oplus \xi)+\rho(T)(\xi) . \\
& \Rightarrow]^{8} \rho(C)(\xi)=\sum \bigcup w_{2 i}^{2}(\xi)+\rho(T)(\xi) .
\end{aligned}
$$

Inserting the polynomial of the corollary from assertion 2, another polynomial in squares is produced:

$$
\Rightarrow \sum \bigcup w_{j}^{2}(\xi)=\rho(T)(\xi) .
$$

As according to [Feshbach, page 513, four lines above "Case 1"], $\rho$ is injective on the torsion elements, there is a local inverse $\left.\rho\right|_{2-\text { Torsion }}{ }^{-1}$ lifting $\rho(T)$ back to $T$.

$$
\begin{aligned}
& \left.\Rightarrow \rho\right|_{2-\text { Torsion }}{ }^{-1}\left(\sum \bigcup w_{j}^{2}(\xi)\right)=T(\xi) . \\
& \left.\Rightarrow^{8} \rho\right|_{2-\text { Torsion }}{ }^{-1}\left(\sum \bigcup w_{2 j}(\xi \oplus \xi)\right)=T(\xi) . \\
& \left.\Rightarrow^{7} \rho\right|_{2-\text { Torsion }}{ }^{-1}\left(\sum \bigcup \rho\left(c_{j}\left(\xi^{\mathbb{C}}\right)\right)\right)=T(\xi) . \\
& \left.\Rightarrow{ }^{n} C(\xi)=\sum \bigcup(-1)^{i} c_{2 i}\left(\xi^{\mathbb{C}}\right)+\left.\rho\right|_{2-\text { Torsion }^{-1}}{ }^{-1} \sum \bigcup \rho\left(c_{j}\left(\xi^{\mathbb{C}}\right)\right)\right) .
\end{aligned}
$$

\footnotetext{
${ }^{6}$ By definition of the Pontrjagin classes.

${ }^{7}$ See [Hatcher, page 83, proposition 3.8] and use $\left(\xi^{\mathbb{C}}\right)_{\mathbb{R}}=\xi \oplus \xi$.

${ }^{8}$ By the Whitney sum axiom and symmetry.
} 
To summarize, unfortunately no extra information about complex vector bundles can be gained introducing topological characteristic classes on "real bone bundles". 


\section{Bibliography}

[P. Alexandroff and H. Hopf] Topologie. First (and only) volume. Corrected reprint, 1974. Springer-Verlag, Berlin, 1935.

[H. Baum] Spin-Strukturen und Dirac-Operatoren über pseudoriemannschen Mannigfaltigkeiten. B.G. Teubner. Leipzig, 1981.

[G.E. Bredon] Topology and geometry. Springer-Verlag, New York, 1993.

[L.E.J. Brouwer] Über Abbildung von Mannigfaltigkeiten. Consultable at

http://www-gdz.sub.uni-goettingen.de/cgi-bin/ digbib.cgi?PPN235181684_0071

Published in volume 71 of the Mathematische Annalen. Editors: Felix Klein, W.v.Dyck, D.Hilbert, O.Blumenthal. Publisher: B.G. Teubner, Leipzig, 1912.

[E.H. Brown, jr.] The Cohomology of $\mathrm{BSO}_{n}$ and $\mathrm{BO}_{n}$ with Integer Coefficients. Stored online:

http://links.jstor.org/sici?sici=0002-9939\% $28198206 \% 2985 \% 3 \mathrm{~A} 2 \% 3 \mathrm{C} 283 \% 3 \mathrm{ATCOAWI} \% 3 \mathrm{E} 2.0 . \mathrm{CO} \% 3 \mathrm{~B} 2-\mathrm{E}$

Proceedings of the American Mathematical Society, Vol. 85, No. 2, pp.283-288, June 1982.

[U. Bunke and T. Schick] Real secondary index theory. Available at: http://www .uni-math.gwdg.de/schick/publ/realgerbe. html

Göttingen, 2003. 
[H. Cartan] Périodicité des groupes d'homotopie stables des groupes classiques, d'après Bott.

17. Démonstration homologique des théorèmes de périodicité de Bott, II: Homologie et cohomologie des groupes classiques et leurs espaces homogènes. Séminaire Henri Cartan, 12e année, Paris, 1959/60.

[M. Feshbach] The Integral Cohomology Rings of the Classifying Spaces of $O(n)$ and $S O(n)$. Indiana University Mathematics Journal, Vol. 32, No. 4, 1983.

[A. Hatcher] Vector Bundles and K-Theory. Available online:

http://www . math. cornell.edu/ hatcher/VBKT/VBpage. html

Version 2.0, January 2003.

[A. Hatcher (AlgTop)] Algebraic Topology. Available online: http://www . math. cornell. edu/ hatcher/AT/ATpage . html

Fourth printing version, last updated July 23, 2006.

[D. Husemoller] Fibre bundles. McGraw-Hill book company, New York etc. Written at Haverford College, 1966.

[H. B. Lawson, jr. and M.-L. Michelsohn] Spin Geometry. Princeton University Press. Princeton, New Jersey, 1989.

[I. Madsen and J. Tornehave] From Calculus to Cohomology. De Rham cohomology and characteristic classes. Cambridge University Press, 1997.

[J.P. May] A Concise Course in Algebraic Topology. The University of Chicago Press. Chicago, 1999.

[J.W. Milnor and J.D. Stasheff] Characteristic classes. Annals of Mathematics Studies, Number 76. Princeton University press and University of Tokyo press. Princeton, New Jersey, 1974.

[R. Stöcker and H. Zieschang] Algebraische Topologie. 2nd edition. Publisher: B.G. Teubner. Stuttgart, 1994. 
[F. Witt] Konforme Invarianten von Lorentz-Flächen. Diplomarbeit, supervised by H.Baum. Until 2006, it was accesible here:

http://page.mi.fu-berlin.de/ fwitt/DA.pdf

Berlin, 2001.

[J.A. Wolf] Spaces of constant curvature. McGraw-Hill book company, Inc. New York etc. Written at the University of California, Berkeley, 1967. 


\section{Index}

almost complex manifold 26

to "appear" 37

Basic requirement 33 cohomology ring of $B \mathcal{O}$ with integral
coefficients 43

"Cutting the equation $c(F \oplus G)$ at the dimension l" 38

Lorentz metric 24

"low situated rest term" 37

Milnor Construction 23

orientability of Riemannian vector bundles: See Application on Riemannian manifolds, 9

orientation bundle 9

pseudo-orthogonal group 13

components of it 15

pseudo-Riemannian manifold 15

its orientability 15

spin structure on it 17

pseudo-Riemannian metric 13

"real bone" bundle 31

H-reduction 7

$\lambda$-reduction 7 spacelike 15

space-orientable 16

spin 17

Subgroup reduction theorem 8 timelike 15

time-orientable 16

time orientation 24

topologically orientable 16

"Transferred stable invariance" 34 